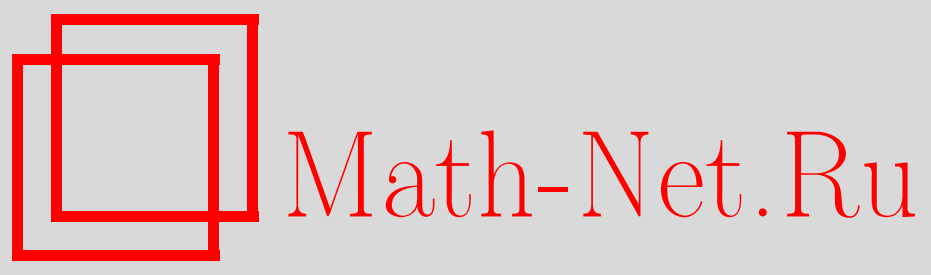

В. В. Волчков, Вит. В. Волчков, Уравнения свертки на многомерных областях и редуцированной группе Гейзенберга, Матем. сб., 2008, том 199, номер 8, 29-60

DOI: https://doi.org/10.4213/sm3945

Использование Общероссийского математического портала Math-Net.Ru подразумевает, что вы прочитали и согласны с пользовательским соглашением http://www . mathnet.ru/rus/agreement

Параметры загрузки:

IP : 54.162 .127 .20

26 апреля 2023 г., 16:48:48

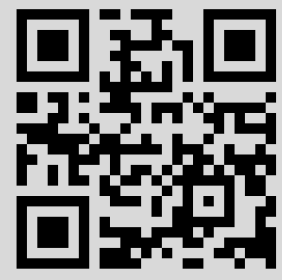




\author{
В. В. Волчков, Вит. В. Волчков
}

\title{
Уравнения свертки на многомерных областях и редуцированной группе Гейзенберга
}

\begin{abstract}
Получены локальные варианты теоремы Брауна-Шрейбера-Тейлора о спектральном анализе в $\mathbb{R}^{n}$ при самых общих предположениях. Это позволило, в частности, доказать эквивалентность глобального и локального свойств Помпейю для компакта $E \subset \mathbb{R}^{n}$ без каких-либо условий на $E$. Установлены точные аналоги этих результатов для систем уравнений свертки на редуцированной группе Гейзенберга $H_{\text {red }}^{n}$. В качестве приложения получена теорема о спектральном анализе для подпространств в $C\left(H_{\mathrm{red}}^{n}\right)$, инвариантных относительно сдвигов и унитарных преобразований, аналоги которой были известны ранее лишь для функций медленного роста.
\end{abstract}

Библиография: 20 названий.

\section{Введение}

Пусть $\mathbb{R}^{n}$ - вещественное евклидово пространство размерности $n$ с евклидовой нормой $|\cdot|, \mathscr{D}^{\prime}(\mathscr{O})$ и $\mathscr{E}^{\prime}(\mathscr{O})$ - пространства распределений и распределений с компактным носителем на открытом множестве $\mathscr{O} \subset \mathbb{R}^{n}$ соответственно. Для $T \in \mathscr{E}^{\prime}\left(\mathbb{R}^{n}\right)$ символом $r(T)$ будем обозначать радиус наименьшего замкнутого шара, содержащего носитель $T$. Для непустого множества индексов $A$ и заданного семейства $\mathscr{T}=\left\{T_{\alpha}\right\}_{\alpha \in A}$ распределений из $\mathscr{E}^{\prime}\left(\mathbb{R}^{n}\right)$ рассмотрим систему уравнений свертки

$$
f * T_{\alpha}=0, \quad \alpha \in A,
$$

с неизвестным $f \in \mathscr{D}^{\prime}\left(\mathbb{R}^{n}\right)$. Для каких $\mathscr{T}$ эта система имеет только нулевое решение? Фундаментальная теорема Л. Шварца о спектральном анализе утверждает, что при $n=1$ для этого необходимо и достаточно, чтобы множество общих нулей преобразований Фурье $\widehat{T}_{\alpha}, \alpha \in A$, было пустым (см., например, [1; теорема 2.11]). При $n \geqslant 2$ точный аналог теоремы Л. Шварца получен Л. Брауном, Б. М. Шрейбером и Б. А. Тейлором [2] в случае, когда все $T_{\alpha}$ являются радиальными. Как показано Д.И. Гуревичем в [3], условие радиальности, вообще говоря, убрать нельзя.

Если все $T_{\alpha}$ радиальны и $r\left(T_{\alpha}\right)<R$ для любого $\alpha \in A$, то естественно изучать систему (1) в шаре $B_{R}=\left\{x \in \mathbb{R}^{n}:|x|<R\right\}$. Эти исследования не только представляют значительный самостоятельный интерес, но и важны для многочисленных приложений в интегральной геометрии, в теории кратных тригонометрических рядов с лакунами, в теории аппроксимации, в комплексном анализе, в уравнениях с частными производными, в теории отображений, сохраняющих меру, и др. (см. [4; часть 5]). К. А. Беренстейн и Р. Гэй установили 
[5; теорема 12], что класс

$$
\mathscr{D}_{\mathscr{T}}^{\prime}\left(B_{R}\right)=\left\{f \in \mathscr{D}^{\prime}\left(B_{R}\right): f * T_{\alpha}=0 \text { в } B_{R-r\left(T_{\alpha}\right)} \forall \alpha \in A\right\}
$$

содержит только нулевое распределение, если существует $\alpha_{0} \in A$ такое, что $T_{\alpha_{0}}$ является гиперболическим,

$$
R>r\left(T_{\alpha_{0}}\right)+\sup _{\alpha \in A} r\left(T_{\alpha}\right)
$$

и $\left\{z \in \mathbb{C}^{n}: \widehat{T}_{\alpha}(z)=0 \forall \alpha \in A\right\}=\varnothing$ (здесь и далее $\mathbb{C}^{n}-$ комплексное евклидово пространство размерности $n$ ). Доказательство этого факта использует соответствующую версию аппроксимационной теоремы Хёрмандера-Напалкова на выпуклых областях в $\mathbb{R}^{n}$ (см. [6; гл. 16], [7; § 20]). Другие методы для изучения системы (1) на ограниченных областях были разработаны в [4]. В частности, для широкого класса семейств $\mathscr{T}$ было найдено описание класса $\mathscr{D}_{\mathscr{T}}^{\prime}\left(B_{R}\right)$ в виде разложений по специальным функциям. Это позволило получить ряд окончательных результатов, усиливающих теорему Беренстейна-Гэя, в которых уточняется оценка (3) и учитывается скорость возможного сближения нулей $\widehat{T}_{\alpha}$ на бесконечности (см. [4; часть 3]). Однако вопрос о справедливости теоремы Беренстейна-Гэя без каких-либо дополнительных ограничений на радиальные распределения $T_{\alpha}$ оставался открытым до настоящего времени.

Другим важным направлением в рассматриваемой тематике является изучение подобных задач на группах Гейзенберга, которые широко используются в современных исследованиях по гармоническому анализу и уравнениям в частных производных (см., например, [8], [9]). Вещественная $(2 n+1)$-мерная группа Гейзенберга $H^{n}$ представляет собой множество $\mathbb{C}^{n} \times \mathbb{R}^{1}$ с законом умножения

$$
(z, t)(w, s)=\left(z+w, t+s+\frac{1}{2} \operatorname{Im}\langle z, w\rangle_{\mathbb{C}}\right), \quad z, w \in \mathbb{C}^{n}, \quad t, s \in \mathbb{R}^{1},
$$

где $\langle z, w\rangle_{\mathbb{C}}$ - эрмитово скалярное произведение в $\mathbb{C}^{n}$. Факторизация $H^{n}$ по подгруппе $\Gamma=\{(0,2 \pi k): k=0, \pm 1, \pm 2, \ldots\}$ порождает редуцированную группу Гейзенберга $H_{\text {red }}^{n}$, функции на которой отождествляются с $2 \pi$-периодическими по $t$ функциями на $H^{n}$ (см. $\left.[9 ; \S 1.2]\right)$. Исследование аналогов системы $(1)$ на $H^{n}$ представляет интерес, по меньшей мере, в силу следующих причин. Во-первых, $H^{n}$ является модельным представителем такого важного класса групп Ли как нильпотентные, и результаты, полученные на $H^{n}$, могут приводить к интересным задачам и гипотезам в общем случае. С другой стороны, $H^{n}$ можно отождествить с помощью преобразования Кэли с единичной сферой $\mathbb{S}^{2 n+1}$ в $\mathbb{C}^{n+1}$, касательные операторы Коши-Римана которой соответствуют основным левоинвариантным векторным полям на $H^{n}$. Тем самым системы типа (1) на $H^{n}$

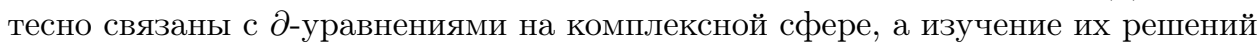
связано с проблемой о голоморфном продолжении внутрь области $\mathscr{O} \subset \mathbb{C}^{n+1}$ функции, заданной на границе $\partial \mathscr{O}$ (см. [10; гл. 5]).

Используя подходящий вариант преобразования Фурье, уравнения свертки на группе $H^{n}$ (соответственно $H_{\text {red }}^{n}$ ) можно свести к искаженным уравнениям свертки на $\mathbb{C}^{n}$ вида

$$
\langle f \times T, \psi\rangle=\left\langle f(z),\left\langle T(w), \psi(z+w) e^{(i / 2) \operatorname{Im}\langle z, w\rangle_{\mathbb{C}}}\right\rangle\right\rangle=0, \quad \psi \in \mathscr{D}\left(\mathbb{C}^{n}\right),
$$


где $f \in \mathscr{D}^{\prime}\left(\mathbb{C}^{n}\right), T \in \mathscr{E}^{\prime}\left(\mathbb{C}^{n}\right), \mathscr{D}\left(\mathbb{C}^{n}\right)$ - пространство финитных бесконечно дифференцируемых функций на $\mathbb{C}^{n}$. Для изучения (4) ранее применялись методы теории идеалов, преобразование Вейля, а также спектральная теория лапласиана Гейзенберга-Кона и оператора $i \frac{\partial}{\partial t}$, развитая Р. Стрихарцем (см. [11], [12] и библиографию к этим работам). Однако полученные при этом результаты были далеки от завершения. В частности, аналоги упомянутой выше теоремы Брауна-Шрейбера-Тейлора были доказаны лишь для функций медленного роста [12; теорема 4.3], а системы искаженных уравнений свертки на ограниченных областях в $\mathbb{C}^{n}$ исследованию не поддавались.

В настоящей работе предлагается принципиально новый подход к задачам указанного типа, который позволяет преодолеть перечисленные выше трудности и состоит в следующем. Для изучения (1) и (4) в $\S 2,7$ вводятся и исследуются операторы, заданные на основных инвариантных подпространствах решений (1) и (4), которые устанавливают гомеоморфизмы между этими подпространствами и пространствами решений специальных одномерных систем уравнений свертки. Их определение существенно опирается на сферическое преобразование в $\mathbb{R}^{n}$ и его аналоги для искаженной свертки, введенные в $\S 6$. Построенные гомеоморфизмы в некотором обобщенном смысле коммутируют с оператором свертки (искаженной свертки), что позволяет свести ряд проблем, связанных с (1) и (4), к одномерному случаю (см. теоремы 5 и 9, (i)). Наконец, для исследования одномерной ситуации важную роль играет теорема 3 о полноте некоторых систем функций (см. также следствие 2). Ее доказательство опирается на свойства целых функций вполне регулярного роста и аналог теоремы Винера-Пэли для аналитических функционалов (см. [6; теорема 15.1.5]).

Основные результаты работы заключаются в следующем.

1. Получен локальный аналог теоремы Беренстейна-Гэя без каких-либо ограничений на радиальные распределения $T_{\alpha}$ и с неулучшаемыми условиями на $R$, ослабляющими (3) (см. теорему 6).

2. Доказана эквивалентность локального и глобального свойства Помпейю в самом общем случае (см. теорему 7 и следствие 3). Отметим, что немногочисленные предшествующие результаты в этом направлении были получены в [13], [4; часть 4] и содержали различные дополнительные требования, связанные с регулярностью границы рассматриваемых множеств.

3. Получены точные аналоги указанных выше евклидовых результатов для систем уравнений свертки на редуцированной группе Гейзенберга (в частности, для систем искаженных уравнений свертки в шаре из $\left.\mathbb{C}^{n}\right)$, см. теоремы 10,12 . В качестве приложения доказана теорема 11 о спектральном анализе для подпространств в $C\left(H_{\text {red }}^{n}\right)$, инвариантных относительно сдвигов и унитарных преобразований, аналоги которой были известны ранее лишь для функций медленного роста (см. [12; теоремы 4.3, 6.2]).

Некоторые промежуточные результаты работы представляют самостоятельный интерес (см. теоремы $3,4,8)$.

Результаты $\S 2$ и теорема 11 получены авторами совместно. Основные результаты $\S \S 3,4$ принадлежат В. В. Волчкову, а $\S \S 5-7-$ Вит. В. Волчкову.

Относительно других аспектов теории уравнений свертки и их приложений см. [6; гл. 16], [7], [14; гл. 5] и библиографию к этим работам. 


\section{§ 1. Основные конструкции и обозначения}

Мы будем использовать следующие стандартные обозначения: $\mathbb{Z}, \mathbb{Z}_{+}, \mathbb{N}$ множества целых, целых неотрицательных и натуральных чисел соответственно; $[\lambda]$ - целая часть числа $\lambda \in \mathbb{R} ;\left(\begin{array}{l}j \\ k\end{array}\right)$ - биномиальные коэффициенты; $\Gamma$ - гамма-функция; $(a)_{j}=\Gamma(a+j) / \Gamma(a), j \in \mathbb{Z}_{+}$, - символ Похгаммера; $\mathbb{Z}_{+}^{n}$ - множество мультииндексов $\alpha=\left(\alpha_{1}, \ldots, \alpha_{n}\right), \alpha_{j} \in \mathbb{Z}_{+}, 1 \leqslant j \leqslant n ;|\alpha|=$ $\alpha_{1}+\cdots+\alpha_{n}$ - длина мультииндекса $\alpha ; z^{\alpha}-$ голоморфный моном в $\mathbb{C}^{n}$, т.е. $z^{\alpha}=z_{1}^{\alpha_{1}} \cdots z_{n}^{\alpha_{n}}$ при $z=\left(z_{1}, \ldots, z_{n}\right) ; \mathscr{D}(\mathscr{O})\left(\mathscr{O}\right.$ - открытое множество в $\left.\mathbb{R}^{n}\right)$ пространство финитных бесконечно дифференцируемых в $\mathscr{O}$ функций; $\mathrm{RA}(\mathscr{O})$ класс вещественно-аналитических в $\mathscr{O}$ функций. Положим также $\mathscr{Z}(f)=$ $\{z \in \mathbb{C}: f(z)=0\}$ для функции $f: \mathbb{C} \rightarrow \mathbb{C}$.

Пусть $T \in \mathscr{E}^{\prime}\left(\mathbb{R}^{n}\right)$. Обозначим

$$
r_{0}(T)=\inf \left\{r>0: \operatorname{supp} T \subset B_{r}\right\},
$$

где supp $T$ - носитель $T$. Преобразование Фурье $\widehat{T}$ распределения $T$ определяется равенством

$$
\widehat{T}(\zeta)=\left\langle T(x), e^{-i\left(x_{1} \zeta_{1}+\cdots+x_{n} \zeta_{n}\right)}\right\rangle, \quad \zeta=\left(\zeta_{1}, \ldots, \zeta_{n}\right) \in \mathbb{C}^{n} .
$$

Если $n=1$ и $T$ четно, положим $\widetilde{T}(z)=\widehat{T}(z), z \in \mathbb{C}$. Ниже мы определим аналог преобразования $\widetilde{T}$ в многомерном случае.

Пусть $n \geqslant 2, \mathbb{S}^{n-1}=\left\{x \in \mathbb{R}^{n}:|x|=1\right\}, \rho$ и $\sigma=\left(\sigma_{1}, \ldots, \sigma_{n}\right)$ - полярные координаты точки $x \in \mathbb{R}^{n}\left(\rho=|x|\right.$, а если $x \in \mathbb{R}^{n} \backslash\{0\}$, то $\left.\sigma=x / \rho\right)$. Обозначим: $\mathscr{H}^{n, k}$ - пространство сферических гармоник степени $k$ на $\mathbb{S}^{n-1}$, рассматриваемое как подпространство $L^{2}\left(\mathbb{S}^{n-1}\right)$ (см. [15; гл. 4]); $d(n, k)-$ размерность $\mathscr{H}^{n, k}$; $\left\{Y_{j}^{k}\right\}, j \in\{1, \ldots, d(n, k)\},-$ фиксированный ортонормированный базис в $\mathscr{H}^{n, k}$. Положим $Y_{1}^{0}(\sigma)=1 / \sqrt{\omega_{n-1}}$ для любого $\sigma \in \mathbb{S}^{n-1}$, где $\omega_{n-1}=n \pi^{n / 2} / \Gamma(1+n / 2)-$ площадь сферы $\mathbb{S}^{n-1}$. Определим $Y_{j}^{k}(x)=\rho^{k} Y_{j}^{k}(\sigma)$ при $x=\rho \sigma \in \mathbb{R}^{n} \backslash\{0\}$. Используя это соотношение, продолжим $Y_{j}^{k}$ до многочлена на $\mathbb{C}^{n}$.

Пусть $T^{n}(\tau), \tau \in O(n),-$ квазирегулярное представление ортогональной группы $O(n)$ в $L^{2}\left(\mathbb{S}^{n-1}\right)$. Тогда $T^{n}(\tau)$ является прямой суммой попарно неэквивалентных неприводимых унитарных представлений $T^{n, k}(\tau)$, действующих на $\mathscr{H}^{n, k}$ (см. [16; гл. 9, $\left.\left.\S 2.1\right]\right)$. Обозначим через $\left\{t_{i, j}^{k}(\tau)\right\}$ матрицу представления $T^{n, k}(\tau)$, т.е.

$$
Y_{j}^{k}\left(\tau^{-1} \sigma\right)=\sum_{i=1}^{d(n, k)} t_{i, j}^{k}(\tau) Y_{i}^{k}(\sigma), \quad \sigma \in \mathbb{S}^{n-1} .
$$

Пусть $\mathscr{O}$ - непустое открытое множество в $\mathbb{R}^{n}$ такое, что $\tau \mathscr{O}=\mathscr{O}$ для любого $\tau \in O(n), L^{1, \operatorname{loc}}(\mathscr{O})$ - класс локально суммируемых в $\mathscr{O}$ функций. Ряд Фурье функции $f \in L^{1, \text { loc }}(\mathscr{O})$ по сферическим гармоникам имеет вид

$$
f(x) \sim \sum_{k=0}^{\infty} \sum_{j=1}^{d(n, k)} f_{k, j}(\rho) Y_{j}^{k}(\sigma), \quad x \in \mathscr{O},
$$

где

$$
f_{k, j}(\rho)=\int_{\mathbb{S}^{n-1}} f(\rho \sigma) \overline{Y_{j}^{k}(\sigma)} d \omega(\sigma)
$$


и $d \omega$ - элемент площади на $\mathbb{S}^{n-1}$. По теореме Фубини функции $f_{k, j}$ корректно определены для почти всех $\rho \in\left\{r>0: S_{r} \subset \mathscr{O}\right\}$, где $S_{r}=\left\{x \in \mathbb{R}^{n}:|x|=r\right\}$.

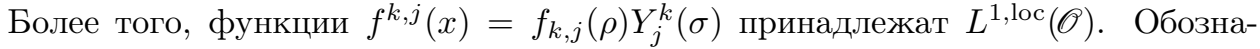
чим через $d \tau$ меру Хаара на $O(n)$, нормированную так, что мера $O(n)$ равна единице. Отображение $f \rightarrow f^{k, j}$ и разложение (5) можно продолжить на распределения $f \in \mathscr{D}^{\prime}(\mathscr{O})$ следующим образом:

$$
\begin{gathered}
\left\langle f^{k, j}, \psi\right\rangle=\left\langle f, d(n, k) \int_{O(n)} \psi\left(\tau^{-1} x\right) t_{j, j}^{k}(\tau) d \tau\right\rangle=\left\langle f, \overline{(\bar{\psi})_{k, j}(\rho)} \overline{Y_{j}^{k}(\sigma)}\right\rangle, \\
\psi \in \mathscr{D}(\mathscr{O}),
\end{gathered}
$$

и

$$
f \sim \sum_{k=0}^{\infty} \sum_{j=1}^{d(n, k)} f^{k, j}
$$

(см. [4; часть 1, п. 5.2]). Отметим, что для любого $f \in \mathscr{D}^{\prime}(\mathscr{O})$ (соответственно, $\left.f \in C^{\infty}(\mathscr{O})\right)$ ряд в правой части $(7)$ сходится к $f$ в $\mathscr{D}^{\prime}(\mathscr{O})$ (соответственно, в $\left.C^{\infty}(\mathscr{O})\right)$.

Действительно, если $f \in C^{\infty}(\mathscr{O})$, то при произвольном $m \in \mathbb{Z}_{+}$имеем (см. $[16 ;$ гл. $9, \S 5$, п. 1 , формула $(7)]$ )

$$
\begin{aligned}
f^{k, j}(x) & =\frac{1}{(k(2-n-k))^{m}} \int_{\mathbb{S}^{n-1}} \Delta_{0}^{m}(f(\rho \eta)) \overline{Y_{j}^{k}(\eta)} d \omega(\eta) Y_{j}^{k}(\sigma) \\
& =\frac{1}{(k(2-n-k))^{m}} \int_{\mathbb{S}^{n-1}}\left(L^{m} f\right)(\rho \eta) \overline{Y_{j}^{k}(\eta)} d \omega(\eta) Y_{j}^{k}(\sigma) \\
& =\frac{1}{(k(2-n-k))^{m}}\left(L^{m} f\right)_{k, j}(\rho) Y_{j}^{k}(\sigma) \\
& =\frac{d(n, k)}{(k(2-n-k))^{m}} \int_{O(n)}\left(L^{m} f\right)\left(\tau^{-1} x\right) \overline{t_{j, j}^{k}(\tau)} d \tau,
\end{aligned}
$$

где $\Delta_{0}$ - оператор Лапласа на $\mathbb{S}^{n-1}$ и

$$
L=\frac{1}{2} \sum_{i, j=1}^{n}\left(x_{i} \frac{\partial}{\partial x_{j}}-x_{j} \frac{\partial}{\partial x_{i}}\right)^{2}
$$

Учитывая, что

$$
d(n, k)=\frac{(2 k+n-2)(n+k-3) !}{(n-2) ! k !}, \quad k \in \mathbb{N}, \quad \int_{O(n)}\left|t_{i, j}^{k}(\tau)\right|^{2} d \tau=\frac{1}{d(n, k)}
$$

(см. [16; гл. $9, \S 2$, п. 5 , формула $(11)$ и гл. $1, \S 4$, п. 3 , формула $(3)])$, получаем сходимость ряда $(7)$ к $f$ в пространстве $C^{\infty}(\mathscr{O})$. Пусть теперь $f \in \mathscr{D}^{\prime}(\mathscr{O})$. Если $\psi \in \mathscr{D}(\mathscr{O})$, то по доказанному выше ряд

$$
\sum_{k=0}^{\infty} \sum_{j=1}^{d(n, k)} \overline{(\bar{\psi})^{k, j}}
$$

сходится к $\psi$ в пространстве $\mathscr{D}(\mathscr{O})$. Этот факт и соотношение (6) влекут сходимость ряда $(7)$ к $f$ в $\mathscr{D}^{\prime}(\mathscr{O})$. 
Для всякого множества $\mathfrak{W}(\mathscr{O}) \subset \mathscr{D}^{\prime}(\mathscr{O})$ положим

$$
\mathfrak{W}_{k, j}(\mathscr{O})=\left\{f \in \mathfrak{W}(\mathscr{O}): f=f^{k, j}\right\} .
$$

Отметим, что $\mathfrak{W}_{0,1}(\mathscr{O})$ совпадает с множеством радиальных распределений из $\mathfrak{W}(\mathscr{O})$. В дальнейшем мы пишем $\mathfrak{W}_{\natural}(\mathscr{O})$ вместо $\mathfrak{W}_{0,1}(\mathscr{O})$. Пусть $R \in(0,+\infty]$. Для любого класса $\mathfrak{W}(-R, R)$ распределений на интервале $(-R, R) \subset \mathbb{R}^{1}$ символом $\mathfrak{W}_{\natural}(-R, R)$ обозначается множество всех четных распределений из $\mathfrak{W}(-R, R)$.

Если $T \in \mathscr{E}_{\natural}^{\prime}\left(\mathbb{R}^{n}\right), r(T)<R \leqslant+\infty$ и $f \in \mathscr{D}^{\prime}\left(B_{R}\right)$, то из $(6)$ следует, что в $B_{R-r(T)}$

$$
(f * T)^{k, j}=f^{k, j} * T \text {. }
$$

Для $\lambda \in \mathbb{C}, \eta \in \mathbb{Z}_{+}$определим

$$
\begin{gathered}
\Phi_{\lambda, \eta, k, j}(x)=\left.2^{n / 2-1} \Gamma\left(\frac{n}{2}\right) \sqrt{\omega_{n-1}} Y_{j}^{k}(x)\left(\frac{\partial}{\partial w}\right)^{\varkappa}\left(\frac{J_{n / 2+k-1}(w|x|)}{(w|x|)^{n / 2+k-1}}\right)\right|_{w=\lambda}, \\
x \in \mathbb{R}^{n},
\end{gathered}
$$

где $J_{\nu}$ - функция Бесселя первого рода порядка $\nu$,

$$
\varkappa=\eta, \text { если } \lambda \neq 0, \quad \text { и } \varkappa=2 \eta, \text { если } \lambda=0 .
$$

Пусть $\Delta$ - оператор Лапласа в $\mathbb{R}^{n}$. Используя дифференциальное уравнение для функций Бесселя [17; п. 7.2, формула (1)] и теорему Эйлера об однородных функциях, из равенства $(9)$ имеем $\left(\Delta+\lambda^{2}\right) \Phi_{\lambda, 0, k, j}=0$. Теперь индукцией по $\eta$ нетрудно проверить, что

$$
\left(\Delta+\lambda^{2}\right)^{\eta} \Phi_{\lambda, \eta, k, j}= \begin{cases}(-\eta)_{\eta}(2 \lambda)^{\eta} \Phi_{\lambda, 0, k, j}, & \text { если } \lambda \neq 0 \\ (-1)^{\eta}(-2 \eta)_{2 \eta} \Phi_{0,0, k, j}, & \text { если } \lambda=0 .\end{cases}
$$

Отсюда

$$
\left(\Delta+\lambda^{2}\right)^{\eta+1} \Phi_{\lambda, \eta, k, j}=0 \quad \text { в } \mathbb{R}^{n} .
$$

Из (9), (10) и интегрального представления Пуассона [17; п. 7.12, формула (8)] находим

$$
\Phi_{\lambda, 0, k, j}(x)=\frac{2^{1-k} \Gamma(n / 2) \sqrt{\omega_{n-1}}}{\sqrt{\pi} \Gamma((n-1) / 2+k)} Y_{j}^{k}(x) \int_{0}^{1}\left(1-t^{2}\right)^{(n-3) / 2+k} \cos (\lambda|x| t) d t .
$$

Далее, из обобщения Гегенбауэра интеграла Пуассона [17; п. 7.8, формула (11)] и формулы Функа-Гекке [17; п. 11.4, формула (23)] получаем

$$
\Phi_{\lambda, 0, k, j}(x)=\frac{\Gamma(n / 2) \sqrt{\omega_{n-1}}}{2 \pi^{n / 2}(i \lambda)^{k}} \int_{\mathbb{S}^{n-1}} e^{i \lambda\langle x, \eta\rangle_{\mathbb{R}}} Y_{j}^{k}(\eta) d \omega(\eta), \quad \lambda \in \mathbb{C} \backslash\{0\},
$$

где $\langle\cdot, \cdot\rangle_{\mathbb{R}}$ - евклидово скалярное произведение в $\mathbb{R}^{n}$. Если $N \in \mathbb{Z}_{+}, R \in(0,+\infty)$ и $\overline{B_{R}}=\left\{x \in \mathbb{R}^{n}:|x| \leqslant R\right\}$, то из этого соотношения следует оценка

$$
\left\|\Phi_{\lambda, 0, k, j}\right\|_{C^{N}\left(\overline{B_{R}}\right)} \leqslant c(1+|\lambda|)^{N-k} e^{R|\operatorname{Im} \lambda|},
$$


где константа $c>0$ не зависит от $\lambda, R$. Кроме того, для $f \in \mathscr{E}_{k, j}^{\prime}\left(\mathbb{R}^{n}\right)$ имеем (см. (6))

$$
\widehat{f}(\zeta)=\frac{2 \pi^{n / 2}}{i^{k} \Gamma(n / 2) \sqrt{\omega_{n-1}}} Y_{j}^{k}(\zeta) \mathscr{F}_{j}^{k}(f)\left(\sqrt{\zeta_{1}^{2}+\cdots+\zeta_{n}^{2}}\right),
$$

где

$$
\mathscr{F}_{j}^{k}(f)(z)=\left\langle f, \overline{\Phi_{\bar{z}, 0, k, j}}\right\rangle, \quad z \in \mathbb{C} .
$$

Если $f \in \mathscr{E}_{\natural}^{\prime}\left(\mathbb{R}^{n}\right)$, то функция $\widetilde{f}(z)=\mathscr{F}_{1}^{0}(f)(z), z \in \mathbb{C}$, называется сферическим преобразованием $f$.

Пусть $T \in \mathscr{E}_{\natural}^{\prime}\left(\mathbb{R}^{n}\right)$. Тогда

$$
\Phi_{\lambda, 0, k, j} * T=\widetilde{T}(\lambda) \Phi_{\lambda, 0, k, j}
$$

по теореме о среднем для собственных функций оператора $\Delta$ (см. (11)). Отсюда

$$
\mathscr{F}_{j}^{k}(f * T)=\mathscr{F}_{j}^{k}(f) \widetilde{T} \quad \text { для всех } f \in \mathscr{E}_{k, j}^{\prime}\left(\mathbb{R}^{n}\right), T \in \mathscr{E}_{\sharp}^{\prime}\left(\mathbb{R}^{n}\right) .
$$

В частности,

$$
\mathscr{F}_{j}^{k}(P(\Delta) f)(z)=P\left(-z^{2}\right) \mathscr{F}_{j}^{k}(f)(z), \quad z \in \mathbb{C},
$$

для любого многочлена $P$.

Далее нам потребуются подобные конструкции для пространства $\mathbb{C}^{n}, n \geqslant 1$. Здесь мы рассмотрим аналоги разложений (5)-(7) для унитарной группы $U(n)$ на $\mathbb{C}^{n}$ и аналог соотношения (8) для искаженной свертки. Построения, связанные с аналогами равенств (9)-(18), содержатся в $\S 5,6$ ниже.

Пусть $\mathscr{H}^{n, p, q}$ - пространство сферических гармоник бистепени $(p, q)$ на $\mathbb{S}^{2 n-1}$ (см. [18; гл. 12]). Как известно [18; теоремы 12.2.7, 12.2.8], квазирегулярное представление группы $U(n)$ в $L^{2}\left(\mathbb{S}^{2 n-1}\right)$ является прямой суммой попарно неэквивалентных неприводимых унитарных представлений, действующих на $\mathscr{H}^{n, p, q}$. Обозначим через $d(n, p, q)$ размерность $\mathscr{H}^{n, p, q}$. Пусть $\left\{S_{l}^{p, q}\right\}$, $l \in\{1, \ldots, d(n, p, q)\},-$ фиксированный ортонормированный базис в $\mathscr{H}^{n, p, q}$. Положим $S_{1}^{0,0}(\sigma)=1 / \sqrt{\omega_{2 n-1}}$ для любого $\sigma \in \mathbb{S}^{2 n-1}$. Всякой функции $f \in L^{1, \text { loc }}(\mathscr{O})$, где $\mathscr{O}$ - непустое открытое $U(n)$-инвариантное множество в $\mathbb{C}^{n}$, соответствует ряд Фурье

$$
f(z) \sim \sum_{p, q=0}^{\infty} \sum_{l=1}^{d(n, p, q)} f_{p, q, l}(\varrho) S_{l}^{p, q}(\sigma), \quad z=\varrho \sigma, \quad \sigma \in \mathbb{S}^{2 n-1},
$$

где

$$
f_{p, q, l}(\varrho)=\int_{\mathbb{S}^{2 n-1}} f(\varrho \sigma) \overline{S_{l}^{p, q}(\sigma)} d \omega(\sigma) .
$$

Разложение (19) можно продолжить на распределения $f \in \mathscr{D}^{\prime}(\mathscr{O})$ следующим образом:

$$
f \sim \sum_{p, q=0}^{\infty} \sum_{l=1}^{d(n, p, q)} f^{p, q, l}
$$


где распределение $f^{p, q, l}$ действует по правилу

$$
\left\langle f^{p, q, l}, \psi\right\rangle=\left\langle f, \overline{(\bar{\psi})_{p, q, l}(\varrho)} \overline{S_{l}^{p, q}(\sigma)}\right\rangle, \quad \psi \in \mathscr{D}(\mathscr{O}) .
$$

Для заданного класса $\mathfrak{W}(\mathscr{O})$ распределений на $\mathscr{O}$ положим

$$
\mathfrak{W}_{p, q, l}(\mathscr{O})=\left\{f \in \mathfrak{W}(\mathscr{O}): f=f^{p, q, l}\right\} .
$$

При $p=q=0, l=1$ будем писать $\mathfrak{W}_{\natural}(\mathscr{O})$ вместо $\mathfrak{W}_{0,0,1}(\mathscr{O})$.

Введем специальный оператор Эрмита (искаженный лапласиан)

$$
\mathfrak{L}=\frac{|z|^{2}}{4} \operatorname{Id}+\sum_{k=1}^{n}\left(z_{k} \frac{\partial}{\partial z_{k}}-\bar{z}_{k} \frac{\partial}{\partial \bar{z}_{k}}-4 \frac{\partial^{2}}{\partial z_{k} \partial \bar{z}_{k}}\right),
$$

где Id - тождественный оператор. Тогда $\mathfrak{L}$ инвариантен относительно искаженных сдвигов $\tau(w) h(z)=h(z+w) e^{\frac{i}{2} \operatorname{Im}\langle z, w\rangle_{\mathbb{C}}}$, т.е.

$$
\mathfrak{L}(\tau(w) h(z))=\tau(w)(\mathfrak{L} h)(z) .
$$

Если $f \in C_{p, q, l}^{2}(\mathscr{O})$, то из $(20)$ непосредственным вычислением получаем соотношение

$$
(\mathfrak{L} f)(z)=\left(\mathfrak{L}_{p, q} f_{p, q, l}\right)(\varrho) S_{l}^{p, q}(\sigma)
$$

где

$$
\mathfrak{L}_{p, q}=-\frac{d^{2}}{d \varrho^{2}}-\frac{2 n-1}{\varrho} \frac{d}{d \varrho}+\left(\frac{(p+q)(2 n+p+q-2)}{\varrho^{2}}+\frac{1}{4} \varrho^{2}+p-q\right) \text { Id } .
$$

Пусть $\mathscr{B}_{R}=\left\{z \in \mathbb{C}^{n}:|z|<R\right\}$. Как и в случае $\mathbb{R}^{n}$, для $T \in \mathscr{E}^{\prime}\left(\mathbb{C}^{n}\right)$ обозначим $r(T)$ радиус наименьшего замкнутого шара в $\mathbb{C}^{n}$, содержащего носитель $T$. Если $T \in \mathscr{E}_{\natural}^{\prime}\left(\mathbb{C}^{n}\right), r(T)<R \leqslant+\infty$ и $f \in \mathscr{D}^{\prime}\left(\mathscr{B}_{R}\right)$, то имеем следующий аналог равенства $(8)$ в $\mathscr{B}_{R-r(T)}$ :

$$
(f \times T)^{p, q, l}=f^{p, q, l} \times T .
$$

В частности, $(P(\mathfrak{L}) f)^{p, q, l}=P(\mathfrak{L}) f^{p, q, l}$ для любого многочлена $P$ (см. предложение 1 , (vii), (ix) ниже).

Для полноты изложения приведем в заключение элементарные свойства искаженной свертки, которые используются далее. Для $z=\left(z_{1}, \ldots, z_{n}\right) \in \mathbb{C}^{n}$ положим $\bar{z}=\left(\bar{z}_{1}, \ldots, \bar{z}_{n}\right)$. Если $T \in \mathscr{D}^{\prime}\left(\mathbb{C}^{n}\right)$, то распределение $T(-\cdot)$ обозначим через $\check{T}$. Пусть также $\delta_{0}-$ мера Дирака в нуле.

ПреДЛОЖЕНИЕ 1. Предположим, что $T_{i} \in \mathscr{D}^{\prime}\left(\mathbb{C}^{n}\right), i=1,2,3$, и хотя бы два из распределений $T_{i}$ имеют компактный носитель.

Тогда:

(i) $\left(\lambda T_{1}+\mu T_{2}\right) \times T_{3}=\lambda\left(T_{1} \times T_{3}\right)+\mu\left(T_{2} \times T_{3}\right), \lambda, \mu \in \mathbb{C}$;

(ii) $\left(T_{1} \times T_{2}\right)^{-}=\check{T}_{1} \times \check{T}_{2}$;

(iii) $\operatorname{supp}\left(T_{1} \times T_{2}\right) \subset \operatorname{supp} T_{1}+\operatorname{supp} T_{2}$;

(iv) $\overline{T_{1} \times T_{2}}=\overline{T_{2}} \times \overline{T_{1}}$;

(v) $\left(T_{1} \times T_{2}\right)(\bar{z})=\left(T_{2} \times T_{1}\right)(z)$, если $T_{i}(z)=T_{i}(\bar{z}), i=1,2$; в частности, $T_{1} \times T_{2}=T_{2} \times T_{1}$ для $T_{1}, T_{2} \in \mathscr{D}_{\natural}^{\prime}\left(\mathbb{C}^{n}\right) ;$ 
(vi) $\left(T_{1} \times T_{2}\right) \times T_{3}=T_{1} \times\left(T_{2} \times T_{3}\right)$;

(vii) $T_{1} \times \delta_{0}=\delta_{0} \times T_{1}=T_{1}$;

(viii) $\left(\tau(w) T_{1}\right) \times T_{2}=\tau(w)\left(T_{1} \times T_{2}\right)$;

(ix) $\mathfrak{L}\left(T_{1} \times T_{2}\right)=T_{1} \times \mathfrak{L} T_{2}, \mathfrak{L}^{*}\left(T_{1} \times T_{2}\right)=\left(\mathfrak{L}^{*} T_{1}\right) \times T_{2},\left(\mathfrak{L} T_{1}\right) \times T_{2}=T_{1} \times \mathfrak{L}^{*} T_{2}$, где $\mathfrak{L}^{*}$ - сопряженный $\kappa$ оператору $\mathfrak{L}$.

ДокАЗАТЕЛЬство. Соотношения (i)-(viii) следуют из определения искаженной свертки (см. (4)) с помощью простых преобразований. Чтобы доказать (ix), достаточно использовать (21).

\section{§ 2. Гомеоморфизмы со свойством трансмутации}

В этом параграфе мы определим оператор, позволяющий свести ряд проблем для уравнений свертки в $\mathbb{R}^{n}$ к одномерному случаю.

Согласно теореме Винера-Пэли [6; теорема 7.3.1], для любого $T \in \mathscr{E}_{\sharp}^{\prime}\left(\mathbb{R}^{n}\right)$ существует распределение $\Lambda(T) \in \mathscr{E}_{\natural}^{\prime}\left(\mathbb{R}^{1}\right)$ такое, что

$$
\widehat{\Lambda(T)}=\widetilde{T}, \quad r(\Lambda(T))=r(T) .
$$

Более того, отображение $\Lambda: \mathscr{E}_{\bigsqcup}^{\prime}\left(\mathbb{R}^{n}\right) \rightarrow \mathscr{E}_{!}^{\prime}\left(\mathbb{R}^{1}\right)$ является биекцией.

Далее на протяжении этого параграфа мы предполагаем, что $n \geqslant 2, k \in \mathbb{Z}_{+}$, $j \in\{1, \ldots, d(n, k)\}$. Для $f \in \mathscr{E}_{k, j}^{\prime}\left(\mathbb{R}^{n}\right)$ и $\psi \in \mathscr{D}\left(\mathbb{R}^{1}\right)$ положим

$$
\left\langle\mathfrak{A}_{k, j}(f), \psi\right\rangle=\frac{\left(\omega_{n-1}\right)^{-1}}{2^{n-2} \Gamma^{2}(n / 2)} \int_{0}^{\infty} \lambda^{n+2 k-1} \mathscr{F}_{j}^{k}(f)(\lambda) \int_{\mathbb{R}^{1}} \psi(t) \cos (\lambda t) d t d \lambda .
$$

Нетрудно убедиться, что $\mathfrak{A}_{k, j}(f) \in \mathscr{D}_{\natural}^{\prime}\left(\mathbb{R}^{1}\right)$. Рассмотрим основные свойства отображения $f \rightarrow \mathfrak{A}_{k, j}(f)$.

Лемма 1. (i) Для $f \in \mathscr{E}_{k, j}^{\prime}\left(\mathbb{R}^{n}\right)$ u $T \in \mathscr{E}_{\natural}^{\prime}\left(\mathbb{R}^{n}\right)$ справедливо соотношение

$$
\mathfrak{A}_{k, j}(f * T)=\mathfrak{A}_{k, j}(f) * \Lambda(T) .
$$

(ii) Пусть $f \in\left(\mathscr{E}_{k, j}^{\prime} \cap C^{N+n+k+2}\right)\left(\mathbb{R}^{n}\right)$ при некотором $N \in \mathbb{Z}_{+}$. Тогда $\mathfrak{A}_{k, j}(f) \in$ $C_{\natural}^{N}\left(\mathbb{R}^{1}\right) u$

$$
f_{k, j}(\varrho)=\frac{2^{1-k} \Gamma(n / 2) \sqrt{\omega_{n-1}}}{\sqrt{\pi} \Gamma((n-1) / 2+k)} \int_{0}^{\varrho} \mathfrak{A}_{k, j}(f)(t) \frac{\left(\varrho^{2}-t^{2}\right)^{(n-3) / 2+k}}{\varrho^{n+k-2}} d t .
$$

(iii) Пусть $f \in \mathscr{E}_{k, j}^{\prime}\left(\mathbb{R}^{n}\right), r \in(0,+\infty]$. Тогда $f=0$ на $B_{r}$ в том и только том случае, когда $\mathfrak{A}_{k, j}(f)=0$ на $(-r, r)$.

ДокАзАтЕльство. Соотношение (26) легко выводится с помощью (25), (24) и (17). Предположим теперь, что выполнены условия пункта (ii). Тогда

$$
\lambda^{n+2 k-1} \mathscr{F}_{j}^{k}(f)(\lambda)=O\left(\lambda^{-N-3}\right)
$$

при $\lambda \rightarrow+\infty\left(\right.$ см. (14)). Отсюда $\mathfrak{A}_{k, j}(f) \in C_{\sharp}^{N}\left(\mathbb{R}^{1}\right)$ и

$$
\mathfrak{A}_{k, j}(f)(t)=\frac{\left(\omega_{n-1}\right)^{-1}}{2^{n-2} \Gamma^{2}(n / 2)} \int_{0}^{\infty} \lambda^{n+2 k-1} \mathscr{F}_{j}^{k}(f)(\lambda) \cos (\lambda t) d \lambda .
$$


Используя [15; гл. 4, теорема 3.10], (28), (12) и формулу обращения для преобразования Фурье, получаем (27). Утверждение (iii) следует из (27) стандартным приемом сглаживания (см. (24), (26) и [4; часть 1, лемма 8.1]).

ЗАмечание 1 . Положив в $(26) T=\Delta \delta_{0}$, находим, что

$$
\mathfrak{A}_{k, j}(\Delta f)=\left(\mathfrak{A}_{k, j}(f)\right)^{\prime \prime} .
$$

Таким образом, $\mathfrak{A}_{k, j}$ обладает трансмутационным свойством (см., например, [19; формула (2.31)]).

Утверждение (iii) леммы 1 позволяет продолжить $\mathfrak{A}_{k, j}$ на пространство $\mathscr{D}_{k, j}^{\prime}\left(B_{R}\right), R \in(0,+\infty]$, по формуле

$$
\left\langle\mathfrak{A}_{k, j}(f), \psi\right\rangle=\left\langle\mathfrak{A}_{k, j}(f \eta), \psi\right\rangle, \quad f \in \mathscr{D}_{k, j}^{\prime}\left(B_{R}\right), \quad \psi \in \mathscr{D}(-R, R),
$$

где $\eta \in \mathscr{D}_{\sharp}\left(B_{R}\right)$ выбирается так, что $\eta=1$ в $B_{r_{0}(\psi)+\varepsilon}$ для некоторого $\varepsilon \in$ $\left(0, R-r_{0}(\psi)\right)$. Тогда $\mathfrak{A}_{k, j}(f) \in \mathscr{D}_{\sharp}^{\prime}(-R, R)$ и $\mathfrak{A}_{k, j}\left(\left.f\right|_{B_{r}}\right)=\left.\mathfrak{A}_{k, j}(f)\right|_{(-r, r)}$ для всех $r \in(0, R]$.

Теорема 1. При $R \in(0,+\infty], N \in \mathbb{Z}_{+} u \nu=N+n+k+2$ имеют место следующие утверждения.

(i) Если $f \in \mathscr{D}_{k, j}^{\prime}\left(B_{R}\right), T \in \mathscr{E}_{\mathfrak{b}}^{\prime}\left(\mathbb{R}^{n}\right)$ u $r(T)<R$, то (26) справедливо на интервале $(r(T)-R, R-r(T))$.

(ii) Пусть $f \in \mathscr{D}_{k, j}^{\prime}\left(B_{R}\right), r \in(0, R]$. Тогда $f=0$ в $B_{r}$ в том и только том cлучае, когда $\mathfrak{A}_{k, j}(f)=0$ на $(-r, r)$.

(iii) Если $f \in C_{k, j}^{\nu}\left(B_{R}\right)$, то $\mathfrak{A}_{k, j}(f) \in C_{\natural}^{N}(-R, R)$ u (27) выполнено при $\varrho \in(0, R)$.

(iv) Отображение $\mathfrak{A}_{k, j}$ непрерьвно из $\mathscr{D}_{k, j}^{\prime}\left(B_{R}\right)$ в $\mathscr{D}_{\natural}^{\prime}(-R, R)$ и из $C_{k, j}^{\nu}\left(B_{R}\right)$ в $C_{\natural}^{N}(-R, R)$.

(v) $\Pi p u \lambda \in \mathbb{C} u \mu \in \mathbb{Z}_{+}$

$$
\mathfrak{A}_{k, j}\left(\Phi_{\lambda, \mu, k, j}\right)=u_{\lambda, \mu}, \quad \text { где } u_{\lambda, \mu}(t)= \begin{cases}\frac{(i t)^{\mu} e^{i \lambda t}+(-i t)^{\mu} e^{-i \lambda t}}{2}, & \lambda \neq 0 \\ (-1)^{\mu} t^{2 \mu}, & \lambda=0 .\end{cases}
$$

ДокАзАтельство. Определение $\mathfrak{A}_{k, j}$ на $\mathscr{D}_{k, j}^{\prime}\left(B_{R}\right)$ и лемма 1 влекут (i)-(iii). $\mathrm{B}(\mathrm{iv})$ предположим сначала, что $f_{q} \in \mathscr{D}_{k, j}^{\prime}\left(B_{R}\right), q=1,2, \ldots$, и $f_{q} \rightarrow 0$ в $\mathscr{D}^{\prime}\left(B_{R}\right)$ при $q \rightarrow+\infty$. Возьмем $\psi \in \mathscr{D}(-R, R)$ и выберем $\eta \in \mathscr{D}_{\mathfrak{b}}\left(B_{R}\right)$ так, что $\eta=1$ в $B_{r_{0}(\psi)+\varepsilon}$ при некотором $\varepsilon \in\left(0, R-r_{0}(\psi)\right)$. Положим $\eta_{\lambda}(x)=\overline{\Phi_{\lambda, 0, k, j}(x)} \eta(x)$. Тогда интеграл

$$
\frac{\left(\omega_{n-1}\right)^{-1}}{2^{n-2} \Gamma^{2}(n / 2)} \int_{0}^{\infty} \lambda^{n+2 k-1} \int_{-R}^{R} \psi(t) \cos (\lambda t) d t \eta_{\lambda}(x) d \lambda
$$

сходится в $\mathscr{D}\left(B_{R}\right)($ см. $(13))$. Обозначая его значение через $\varphi(x)$, имеем

$$
\left\langle\mathfrak{A}_{k, j}\left(f_{q}\right), \psi\right\rangle=\frac{\left(\omega_{n-1}\right)^{-1}}{2^{n-2} \Gamma^{2}(n / 2)} \int_{0}^{\infty} \lambda^{n+2 k-1} \int_{-R}^{R} \psi(t) \cos (\lambda t) d t\left\langle f_{q}, \eta_{\lambda}\right\rangle d \lambda=\left\langle f_{q}, \varphi\right\rangle,
$$


откуда $\mathfrak{A}_{k, j}\left(f_{q}\right) \rightarrow 0$ в $\mathscr{D}^{\prime}(-R, R)$. Пусть теперь $f_{q} \in C_{k, j}^{\nu}\left(B_{R}\right), q=1,2, \ldots$, и $f_{q} \rightarrow 0$ в $C^{\nu}\left(B_{R}\right)$. Зафиксируем $r \in(0, R)$. Снова выберем $\eta \in \mathscr{D}_{\sharp}\left(B_{R}\right)$ такую, что $\eta=1$ в $B_{r+\varepsilon}$ при некотором $\varepsilon \in(0, R-r)$. Используя $(28),(11)$ и (13), получаем $\left\|\mathfrak{A}_{k, j}\left(\eta f_{q}\right)\right\|_{C^{N}[-r, r]} \leqslant c\left\|\eta f_{q}\right\|_{C^{\nu}(E)}$, где $E=\operatorname{supp} \eta$ и константа $c>0$ не зависит от $q$. В силу этого и утверждения (iі) получаем, что $\lim _{q \rightarrow+\infty}\left\|\mathfrak{A}_{k, j}\left(f_{q}\right)\right\|_{C^{N}[-r, r]}=0$. Таким образом, $\mathfrak{A}_{k, j}\left(f_{q}\right) \rightarrow 0$ в $C^{N}(-R, R)$ и (iv) установлено. Далее, благодаря (30), (27) и (12),

$$
\int_{0}^{\varrho}\left(\mathfrak{A}_{k, j}\left(\Phi_{\lambda, 0, k, j}\right)(t)-\cos (\lambda t)\right)\left(\varrho^{2}-t^{2}\right)^{(n-3) / 2+k} d t=0
$$

для всех $\varrho \in(0, R)$. Поэтому $\mathfrak{A}_{k, j}\left(\Phi_{\lambda, 0, k, j}\right)(t)=\cos (\lambda t), t \in(-R, R)$ (см., например, [20; гл. 1, доказательство теоремы 2.6]). Дифференцируя последнее равенство по $\lambda$, приходим к (v).

Из утверждения (ii) теоремы 1 видно, что отображение $\mathfrak{A}_{k, j}$ инъективно. Наша дальнейшая цель - найти обратный оператор $\mathfrak{A}_{k, j}^{-1}$. Для $F \in \mathscr{E}_{\natural}^{\prime}\left(\mathbb{R}^{1}\right)$ положим

$$
\begin{aligned}
\left\langle\mathfrak{B}_{k, j}(F), w\right\rangle & =\frac{1}{\pi} \int_{0}^{\infty} \widehat{F}(\lambda) \mathscr{F}_{j}^{k}\left(\overline{(\bar{w})_{k, j}(\varrho)} Y_{j}^{k}(\sigma)\right)(\lambda) d \lambda \\
& =\frac{1}{\pi} \int_{0}^{\infty} \widehat{F}(\lambda)\left\langle w, \Phi_{\lambda, 0, k, j}\right\rangle d \lambda, \quad w \in \mathscr{D}\left(\mathbb{R}^{n}\right) .
\end{aligned}
$$

Используя (11), (13) и теорему Винера-Пэли, делаем вывод, что $\mathfrak{B}_{k, j}(F) \in$ $\mathscr{D}_{k, j}^{\prime}\left(\mathbb{R}^{n}\right)$ и отображение $\mathfrak{B}_{k, j}: \mathscr{E}_{\sharp}^{\prime}\left(\mathbb{R}^{1}\right) \rightarrow \mathscr{D}_{k, j}^{\prime}\left(\mathbb{R}^{n}\right)$ является непрерывным.

Лемма 2. (i) При $F \in \mathscr{E}_{\natural}^{\prime}\left(\mathbb{R}^{1}\right)$ u $T \in \mathscr{E}_{\natural}^{\prime}\left(\mathbb{R}^{n}\right)$ имеем

$$
\mathfrak{B}_{k, j}(F) * T=\mathfrak{B}_{k, j}(F * \Lambda(T)) .
$$

(ii) Пусть $F \in\left(\mathscr{E}_{\natural}^{\prime} \cap C^{s}\right)\left(\mathbb{R}^{1}\right)$ для некоторого $s \geqslant 2$. Тогда $\mathfrak{B}_{k, j}(F) \in$ $C_{k, j}^{s+k-2}\left(\mathbb{R}^{n}\right) u$

$$
\left(\mathfrak{B}_{k, j}(F)\right)(x)=\frac{2^{1-k} \Gamma(n / 2) \sqrt{\omega_{n-1}}}{\sqrt{\pi} \Gamma((n-1) / 2+k)} \int_{0}^{\varrho} F(t) \frac{\left(\varrho^{2}-t^{2}\right)^{(n-3) / 2+k}}{\varrho^{n+k-2}} d t Y_{j}^{k}(\sigma) .
$$

(iii) Пусть $F \in \mathscr{E}_{\llcorner}^{\prime}\left(\mathbb{R}^{1}\right)$ u $r \in(0,+\infty]$. Тогда $F=0$ на $(-r, r)$ в том и только том случае, когда $\mathfrak{B}_{k, j}(F)=0$ в $B_{r}$.

ДокАзАТЕЛЬСтво. Возьмем произвольную $w \in \mathscr{D}\left(\mathbb{R}^{n}\right)$. Учитывая $(24),(11)$ и (16), получаем

$$
\begin{aligned}
\left\langle\mathfrak{B}_{k, j}(F) * T, w\right\rangle & =\left\langle\mathfrak{B}_{k, j}(F), w * T\right\rangle=\frac{1}{\pi} \int_{0}^{\infty} \widehat{F}(\lambda)\left\langle w, \Phi_{\lambda, 0, k, j} * T\right\rangle d \lambda \\
& =\frac{1}{\pi} \int_{0}^{\infty} \widehat{F}(\lambda) \widehat{\Lambda(T)}(\lambda)\left\langle w, \Phi_{\lambda, 0, k, j}\right\rangle d \lambda=\left\langle\mathfrak{B}_{k, j}(F * \Lambda(T)), w\right\rangle,
\end{aligned}
$$

что доказывает (33). Для проверки (ii) заметим, что при $F \in\left(\mathscr{E}_{\natural}^{\prime} \cap C^{s}\right)\left(\mathbb{R}^{1}\right)$, $s \geqslant 2$,

$$
\mathfrak{B}_{k, j}(F)(x)=\frac{1}{\pi} \int_{0}^{\infty} \widehat{F}(\lambda) \Phi_{\lambda, 0, k, j}(x) d \lambda, \quad x \in \mathbb{R}^{n}
$$


(см. (32) и (13)). Теперь, применяя (12) и формулу обращения для косинуспреобразования Фурье, приходим к (ii). Утверждение (iii) следует из (ii) посредством сглаживания (см. (24) и (33)).

Ввиду утверждения (iii) леммы 2 отображение $\mathfrak{B}_{k, j}$ можно продолжить на пространство $\mathscr{D}_{\natural}^{\prime}(-R, R), R \in(0,+\infty]$, с помощью равенства

$$
\left\langle\mathfrak{B}_{k, j}(F), w\right\rangle=\left\langle\mathfrak{B}_{k, j}(F \eta), w\right\rangle, \quad F \in \mathscr{D}_{\natural}^{\prime}(-R, R), \quad w \in \mathscr{D}\left(B_{R}\right),
$$

где $\eta \in \mathscr{D}_{\mathfrak{\natural}}(-R, R)$ и $\eta=1$ на $\left(-r_{0}(w)-\varepsilon, r_{0}(w)+\varepsilon\right)$ при некотором $\varepsilon \in$ $\left(0, R-r_{0}(w)\right)$. При этом $\mathfrak{B}_{k, j}(F) \in \mathscr{D}_{k, j}^{\prime}\left(B_{R}\right)$ и $\mathfrak{B}_{k, j}\left(\left.F\right|_{(-r, r)}\right)=\left.\mathfrak{B}_{k, j}(F)\right|_{B_{r}}$ для всех $r \in(0, R]$.

Теорема 2. Для $R \in(0,+\infty], s \in\{2,3, \ldots\}$ справедливъ следующие утверждения.

(i) Пусть $F \in \mathscr{D}_{\natural}^{\prime}(-R, R), T \in \mathscr{E}_{\natural}^{\prime}\left(\mathbb{R}^{n}\right)$ u $r(T)<R$. Тогда (33) выполнено $8 B_{R-r(T)}$.

(ii) Пусть $F \in \mathscr{D}_{\llcorner}^{\prime}(-R, R), r \in(0, R]$. Тогда $F=0$ на $(-r, r)$ в том и только том случае, когда $\mathfrak{B}_{k, j}(F)=0$ в $B_{r}$.

(iii) Если $F \in C_{\natural}^{s}(-R, R)$, то $\mathfrak{B}_{k, j}(F) \in C_{k, j}^{s+k-2}\left(B_{R}\right)$ u (34) справедливо в $B_{R} \backslash\{0\}$.

(iv) Отображение $\mathfrak{B}_{k, j}$ непреръьно из $\mathscr{D}_{\natural}^{\prime}(-R, R)$ в $\mathscr{D}_{k, j}^{\prime}\left(B_{R}\right)$ и из $C_{\natural}^{s}(-R, R)$ в $C_{k, j}^{s+k-2}\left(B_{R}\right)$.

(v) Eсли $F \in \mathscr{D}_{\mathfrak{\natural}}^{\prime}(-R, R)$, mo $\mathfrak{A}_{k, j}\left(\mathfrak{B}_{k, j}(F)\right)=F$.

ДокАзАтельство. Получается незначительной модификацией рассуждений из теоремы 1 (см. лемму 2).

СлеДСТВИЕ 1. Для любого $R \in(0,+\infty]$ преобразование $\mathfrak{A}_{k, j}$ устанавливает гомеоморфизм между:

(i) $\mathscr{D}_{k, j}^{\prime}\left(B_{R}\right)$ u $\mathscr{D}_{\mathfrak{b}}^{\prime}(-R, R)$;

(ii) $C_{k, j}^{\infty}\left(B_{R}\right) u C_{\natural}^{\infty}(-R, R)$.

Кроме того, $\mathfrak{A}_{k, j}^{-1}=\mathfrak{B}_{k, j}$.

ДоказАтЕЛьство. Непосредственно вытекает из теорем 1 и 2.

\section{§ 3. Вспомогательные результаты о полноте некоторых систем функций}

Пусть $T \in \mathscr{E}^{\prime}\left(\mathbb{R}^{1}\right), T \neq 0$ и $\operatorname{supp} T \subset[-r(T), r(T)]$. По теореме Винера-Пэли [6; теорема 7.3.1]

$$
|\widehat{T}(z)| \leqslant \gamma_{1}(1+|z|)^{\gamma_{2}} e^{r(T)|\operatorname{Im} z|}, \quad z \in \mathbb{C}
$$

где $\gamma_{1}, \gamma_{2}>0$ не зависят от $z$.

Пусть $\mathscr{Z}(\widehat{T}) \neq \varnothing, \lambda \in \mathscr{Z}(\widehat{T}), \eta \in\{0, \ldots, m(\lambda, T)-1\}$, где $m(\lambda, T)-$ кратность нуля $\lambda$ функции $\widehat{T}$. Из (37) и теоремы Винера-Пэли следует, что существует $T^{\lambda, \eta} \in \mathscr{E}^{\prime}\left(\mathbb{R}^{1}\right)$ такое, что

$$
\operatorname{supp} T^{\lambda, \eta} \subset[-r(T), r(T)], \quad \widehat{T}^{\lambda, \eta}(z)(z-\lambda)^{\eta+1}=\widehat{T}(z), \quad z \in \mathbb{C} .
$$


Из (38) находим

$$
\left(-i \frac{d}{d t}-\lambda\right)^{\eta+1} T^{\lambda, \eta}=T
$$

Tеорема 3. Пусть $T \in\left(\mathscr{E}^{\prime} \cap L^{1}\right)\left(\mathbb{R}^{1}\right), r(T)>0, \operatorname{supp} T \subset[-r(T), r(T)]$, $f \in L^{1}[-r(T), r(T)] u$

$$
\left\langle T^{\lambda, \eta}, f\right\rangle=0 \text { для всех } \lambda \in \mathscr{Z}(\widehat{T}), \eta \in\{0, \ldots, m(\lambda, T)-1\} .
$$

Тогда $f=0$.

ДокАЗАТЕЛЬСтво. Для краткости положим $r=r(T)$. Рассмотрим целую функцию

$$
u(z)=e^{-i r z} \int_{-r}^{r} f(x) \int_{-r}^{x} T(t) e^{i z(x-t)} d t d x, \quad z \in \mathbb{C} .
$$

Продолжая $f(x)$ нулем при $x \in \mathbb{R}^{1} \backslash[-r, r]$, имеем

$$
\begin{aligned}
u(z) & =e^{-i r z} \int_{-r}^{r} f(-x) \int_{-r}^{-x} T(t) e^{-i z(x+t)} d t d x \\
& =e^{-i r z} \widehat{f}(-z) \widehat{T}(z)-e^{-i r z} \int_{-r}^{r} f(-x) \int_{-x}^{r} T(t) e^{-i z(x+t)} d t d x \\
& =e^{-i r z} \widehat{f}(-z) \widehat{T}(z)-e^{-2 i r z} v(z),
\end{aligned}
$$

где

$$
v(z)=e^{i r z} \int_{-r}^{r} f(-x) \int_{-r}^{x} T(-t) e^{i z(t-x)} d t d x .
$$

Равенства (41) и (43) показывают, что

$$
|u(z)|+|v(z)| \leqslant c_{1} e^{r|z|}, \quad z \in \mathbb{C},
$$

где $c_{1}>0$ не зависит от $z$. Используя (39), получаем

$$
T^{\lambda, \eta}(t)=\frac{i^{\eta+1}}{\eta !} \int_{-r}^{t} T(\xi)(t-\xi)^{\eta} e^{i \lambda(t-\xi)} d \xi, \quad t \in \mathbb{R}^{1} .
$$

Тогда из (40) и (41) имеем $u^{(\eta)}(\lambda)=0$ для всех $\lambda, \eta$. Следовательно, функции $u_{1}=u / \widehat{T}$ и $u_{2}=v / \widehat{T}$ являются целыми (см. (42)). Соотношение (42) дает

$$
u_{1}(z) e^{i r z}+u_{2}(z) e^{-i r z}=\widehat{f}(-z), \quad z \in \mathbb{C} .
$$

Согласно [7; следствие 15.22], (44) и (37), для любого $\varepsilon>0$ существует $c_{2}=$ $c_{2}(\varepsilon)>0$ такая, что

$$
\left|u_{1}(z)\right|+\left|u_{2}(z)\right| \leqslant c_{2} e^{\varepsilon|z|} \quad \text { для всех } z \in \mathbb{C} .
$$

Далее, пусть

$$
\begin{gathered}
\alpha \in(0, r), \quad \beta \in\left(0, \frac{r-\alpha}{2}\right), \\
E_{1, \beta}=\{z \in \mathbb{C}:|z-r| \leqslant \beta\}, \quad E_{2, \beta}=\{z \in \mathbb{C}:|z+r| \leqslant \beta\} .
\end{gathered}
$$


Из оценки (47) следует существование мер $\mu_{1}$ и $\mu_{2}$ на $\mathbb{C}$ таких, что supp $\mu_{j} \subset E_{j, \beta}$, $j \in\{1,2\}$, и $\mu_{1}\left(e^{-i \zeta(\cdot-r)}\right)+\mu_{2}\left(e^{-i \zeta(\cdot+r)}\right)=\widehat{f}(-\zeta)$ для всех $\zeta \in \mathbb{C}$ (см. [6; теорема 15.1.5] и (46)). Поскольку $\zeta$ является произвольным,

$$
\mu_{1}(w(\cdot-r))+\mu_{2}(w(\cdot+r))=\int_{-r}^{r} f(-x) w(x) d x
$$

для любой целой функции $w: \mathbb{C} \rightarrow \mathbb{C}$. Таким образом,

$$
\left|\int_{-r}^{r} f(-x) w(x) d x\right| \leqslant c_{3} \max _{z \in E_{\beta}}|w(z)|,
$$

где $E_{\beta}=E_{1, \beta} \cup E_{2, \beta}$ и $c_{3}>0$ не зависит от $w$. Теперь определим

$$
\varphi_{k}(z)=\frac{k}{\sqrt{\pi}} e^{-(k z)^{2}}, \quad k \in \mathbb{N}, \quad z \in \mathbb{C} .
$$

Тогда $\varphi_{k}(z) \geqslant 0$ для $z \in \mathbb{R}^{1}$ и

$$
\int_{\mathbb{R}^{1}} \varphi_{k}(t) d t=1
$$

Полагая $w(z)=\varphi_{k}(z-\gamma), \gamma \in(-\alpha, \alpha)$, из (48) и (49) видим, что

$$
\left|\left(f * \varphi_{k}\right)(\gamma)\right| \leqslant c_{3} \exp \left(-\frac{k^{2}}{4}\left((r-\alpha)^{2}-4 \beta^{2}\right)\right),
$$

где $c_{3}>0$ не зависит от $k$. Устремив $k$ к бесконечности, отсюда получаем, что $f=0$ на $(-\alpha, \alpha)$. В силу произвольности $\alpha \in(0, r)$ это завершает доказательство.

Следствие 2. Пусть $T \in \mathscr{E}^{\prime}\left(\mathbb{R}^{1}\right), T \neq 0, \operatorname{supp} T \subset[-r(T), r(T)] u \mathscr{Z}(\widehat{T}) \neq \varnothing$. Пусть также $R>r(T), f \in \mathscr{D}^{\prime}(-R, R) u$

$$
f * T^{\lambda, m(\lambda, T)-1}=0 \quad \text { в }(-R+r(T), R-r(T)) \quad \text { для всех } \lambda \in \mathscr{Z}(\widehat{T}) .
$$

Тогда $f=0$.

ДокАЗАТЕЛЬСтво. При $r(T)=0$ утверждение следует из $(38)$ и [4; часть 3 , теорема 1.7]. Пусть $r(T)>0$. Из (50) и (39) получаем, что $f * T^{\lambda, \eta}=0$ при всех $\lambda \in \mathscr{Z}(\widehat{T}), \eta \in\{0, \ldots, m(\lambda, T)-1\}$. Кроме того, в рассматриваемом случае множество $\mathscr{Z}(\widehat{T})$ является бесконечным и существуют многочлены $p_{1}, p_{2}$ такие, что $\mathscr{Z}\left(p_{1}\right) \cap \mathscr{Z}\left(p_{2}\right)=\varnothing$ и

$$
p_{1}\left(-i \frac{d}{d t}\right) U_{1}=p_{2}\left(-i \frac{d}{d t}\right) U_{2}=T
$$

для некоторых $U_{1}, U_{2} \in\left(\mathscr{E}^{\prime} \cap L^{1}\right)\left(\mathbb{R}^{1}\right) . \quad$ Пусть $j \in\{1,2\}, \lambda \in \mathscr{Z}\left(\widehat{U}_{j}\right), \eta \in$ $\left\{0, \ldots, m\left(\lambda, U_{j}\right)-1\right\}$. Тогда $r\left(U_{j}\right)=r(T), \lambda \in \mathscr{Z}(\widehat{T}), m\left(\lambda, U_{j}\right) \leqslant m(\lambda, T)$ и

$$
p_{j}\left(-i \frac{d}{d t}\right) U_{j}^{\lambda, \eta}=T^{\lambda, \eta} .
$$


Таким образом,

$$
p_{j}\left(-i \frac{d}{d t}\right)\left(f * \varphi * U_{j}^{\lambda, \eta}\right)=0
$$

для любой $\varphi \in \mathscr{D}(-R+r(T), R-r(T))$. В силу произвольности $\varphi$ по теореме 3

$$
p_{j}\left(-i \frac{d}{d t}\right) f=0 \quad \text { на }(-R, R) .
$$

Поскольку $\mathscr{Z}\left(p_{1}\right) \cap \mathscr{Z}\left(p_{2}\right)=\varnothing$, отсюда следует требуемое утверждение.

До конца этого параграфа мы предполагаем, что $n \geqslant 1, T \in \mathscr{E}_{\natural}^{\prime}\left(\mathbb{R}^{n}\right), T \neq 0$ и $\mathscr{Z}(\widetilde{T}) \neq \varnothing$. Используя теорему Винера-Пэли, для любого $\lambda \in \mathscr{Z}(\widetilde{T})$ определим $T_{(\lambda)} \in \mathscr{E}_{\natural}^{\prime}\left(\mathbb{R}^{n}\right)$ по формуле

$$
\widetilde{T}_{(\lambda)}(z)= \begin{cases}\frac{\widetilde{T}(z)}{\left(z^{2}-\lambda^{2}\right)^{m(\lambda, T)}}, & \text { если } \lambda \neq 0, \\ \frac{\widetilde{T}(z)}{z^{m(0, T)}}, & \text { если } \lambda=0 \in \mathscr{Z}(\widetilde{T}),\end{cases}
$$

где $m(\lambda, T)$ - кратность нуля $\lambda$ функции $\widetilde{T}$.

Tеорема 4. Пусть $r(T)<R \leqslant+\infty, f \in \mathscr{D}^{\prime}\left(B_{R}\right) u$

$$
f * T_{(\lambda)}=0 \quad \text { в } B_{R-r(T)} \quad \text { для всех } \lambda \in \mathscr{Z}(\widetilde{T}) .
$$

Тогда $f=0$.

ДокАЗАтельство. Пусть сначала $n=1$. Тогда из (51), (52) и (38) следует равенство (50). Используя следствие 2 , получаем $f=0$. В случае $n \geqslant 2$ достаточно доказать, что $f^{k, j}=0$ для всех $k \in \mathbb{Z}_{+}, j \in\{1, \ldots, d(n, k)\}$ (см. (7)). Если $\lambda \in \mathscr{Z}(\widetilde{T})$, то

$$
\mathfrak{A}_{k, j}\left(f^{k, j}\right) * \Lambda\left(T_{(\lambda)}\right)=\mathfrak{A}_{k, j}\left(f^{k, j} * T_{(\lambda)}\right)=0
$$

ввиду (52), (6) и теоремы 1, (i). Осталось применить следствие 2 и утверждение (ii) теоремы 1.

\section{§ 4. Системы уравнений свертки на ограниченных областях в $\mathbb{R}^{n}$}

Пусть $\mathfrak{T}\left(\mathbb{R}^{n}\right)$ - совокупность всех семейств $\mathscr{T}=\left\{T_{\alpha}\right\}_{\alpha \in A}$, где $A-$ фиксированное непустое множество индексов и $T_{\alpha} \in \mathscr{E}_{\natural}^{\prime}\left(\mathbb{R}^{n}\right)$ при всех $\alpha \in A$. Для $\mathscr{T} \in \mathfrak{T}\left(\mathbb{R}^{n}\right)$ положим

$$
\mathscr{Z}_{\mathscr{T}}=\bigcap_{\alpha \in A} \mathscr{Z}\left(\widetilde{T}_{\alpha}\right), \quad \Lambda(\mathscr{T})=\left\{\Lambda\left(T_{\alpha}\right)\right\}_{\alpha \in A}
$$

(см. (24)). Отметим, что $\Lambda(\mathscr{T}) \in \mathfrak{T}\left(\mathbb{R}^{1}\right)$. Пусть также $C_{\mathscr{T}}^{\infty}\left(B_{R}\right)=\left(\mathscr{D}_{\mathscr{T}}^{\prime} \cap\right.$ $\left.C^{\infty}\right)\left(B_{R}\right)$, где класс $\mathscr{D}_{\mathscr{T}}^{\prime}\left(B_{R}\right)$ определяется равенством $(2)$ при любом $n \geqslant 1$.

Tеорема 5. Пусть $n \geqslant 2, \mathscr{T} \in \mathfrak{T}_{\natural}\left(\mathbb{R}^{n}\right)$ u $r\left(T_{\alpha}\right)<R \leqslant+\infty$ для любого $\alpha \in A$. Тогда при фиксированных $k \in \mathbb{Z}_{+}, j \in\{1, \ldots, d(n, k)\}$ следующие утвержсденя эквивалентны: 
(i) $\mathscr{D}_{\mathscr{T}}^{\prime}\left(B_{R}\right)=\{0\}$;

(ii) $\left(\mathscr{D}_{\mathscr{T}}^{\prime} \cap \mathscr{D}_{k, j}^{\prime}\right)\left(B_{R}\right)=\{0\}$;

(iii) $\left(\mathscr{D}_{\Lambda(\mathscr{T})}^{\prime} \cap \mathscr{D}_{\sharp}^{\prime}\right)(-R, R)=\{0\}$.

То же самое верно, если множества $\mathscr{D}_{\mathscr{T}}^{\prime}\left(B_{R}\right),\left(\mathscr{D}_{\mathscr{T}}^{\prime} \cap \mathscr{D}_{k, j}^{\prime}\right)\left(B_{R}\right),\left(\mathscr{D}_{\Lambda(\mathscr{T})}^{\prime} \cap\right.$ $\left.\mathscr{D}_{\sharp}^{\prime}\right)(-R, R)$ заменить на $C_{\mathscr{T}}^{\infty}\left(B_{R}\right),\left(C_{\mathscr{T}}^{\infty} \cap \mathscr{D}_{k, j}^{\prime}\right)\left(B_{R}\right),\left(C_{\Lambda(\mathscr{T})}^{\infty} \cap \mathscr{D}_{\natural}^{\prime}\right)(-R, R)$ coответственно.

Доказательство. Импликация (i) $\Longrightarrow$ (ii) очевидна. Далее, из утверждения (i) теоремы 2 и следствия 1 видно, что (ii) влечет (iii). Пусть теперь выполнено (iii) и предположим, что $f \in \mathscr{D}_{\mathscr{T}}^{\prime}\left(B_{R}\right)$. В силу (8) и теоремы $1,(\mathrm{i})$

$$
f^{k, j} \in \mathscr{D}_{\mathscr{T}}^{\prime}\left(B_{R}\right), \quad \mathfrak{A}_{k, j}\left(f^{k, j}\right) \in\left(\mathscr{D}_{\Lambda(\mathscr{T})}^{\prime} \cap \mathscr{D}_{\natural}^{\prime}\right)(-R, R)
$$

для всех $k \in \mathbb{Z}_{+}, j \in\{1, \ldots, d(n, k)\}$. В сочетании с (iii) и следствием 1 это дает $f^{k, j}=0$ для всех $k, j$. Поэтому $f=0$ и (i) является следствием (iii). Чтобы доказать тот же результат для классов $C_{\mathscr{T}}^{\infty}\left(B_{R}\right),\left(C_{\mathscr{T}}^{\infty} \cap \mathscr{D}_{k, j}^{\prime}\right)\left(B_{R}\right)$ и $\left(C_{\Lambda(\mathscr{T})}^{\infty} \cap \mathscr{D}_{\natural}^{\prime}\right)(-R, R)$, достаточно повторить рассуждения выше с использованием утверждения (i) теоремы 2 и следствия 1.

Далее, для любого семейства $\mathscr{T}=\left\{T_{\alpha}\right\}_{\alpha \in A}$ распределений класса $\mathscr{E}^{\prime}\left(\mathbb{R}^{n}\right)$ положим

$$
r_{*}(\mathscr{T})=\inf _{\alpha \in A} r\left(T_{\alpha}\right), \quad r^{*}(\mathscr{T})=\sup _{\alpha \in A} r\left(T_{\alpha}\right), \quad R_{\mathscr{T}}=r_{*}(\mathscr{T})+r^{*}(\mathscr{T}) .
$$

TеОрема 6. Пусть $\mathscr{T} \in \mathfrak{T}\left(\mathbb{R}^{n}\right)$ и $\mathscr{Z}_{\mathscr{T}}=\varnothing$. Тогда имеют место следующие утверждения:

(i) если $r_{*}(\mathscr{T})+r\left(T_{\alpha}\right)<R \leqslant+\infty$ для всех $\alpha \in A$, mо $\mathscr{D}_{\mathscr{T}}^{\prime}\left(B_{R}\right)=\{0\}$;

(ii) если $r\left(T_{\alpha}\right)<r^{*}(\mathscr{T})<+\infty$ для всех $\alpha \in A$ u $R_{\mathscr{T}} \leqslant R \leqslant+\infty$, mо $\mathscr{D}_{\mathscr{T}}^{\prime}\left(B_{R}\right)=\{0\}$.

Кроме того, при $n=1$ утверждения $(i)$ и (ii) верны для любого семейства $\mathscr{T}=\left\{T_{\alpha}\right\}_{\alpha \in \text { A }}$ распределений из $\mathscr{E}^{\prime}\left(\mathbb{R}^{1}\right)$ такого, что $\bigcap_{\alpha \in A} \mathscr{Z}\left(\widehat{T}_{\alpha}\right)=\varnothing$.

Отметим, что условия на $R$ в теореме 6 в общем случае ослабить нельзя (см. [4; часть 2, теорема $1.6(5)]$ ).

Для доказательства теоремы 6 потребуется следующая

Лемма 3. Пусть $U$ и $V$ - ненулевые распределения класса $\mathscr{E}^{\prime}\left(\mathbb{R}^{1}\right)$,

$$
\operatorname{supp} U \subset[-r(U), r(U)], \quad \operatorname{supp} V \subset[-r(V), r(V)], \quad \lambda \in \mathscr{Z}(\widehat{U}) \backslash \mathscr{Z}(\widehat{V}) .
$$

Предположим, что $R>r(U)+r(V), f \in \mathscr{D}^{\prime}(-R, R), f * U=0$ на $(-R+r(U)$, $R-r(U))$ u $f * V=0$ на $(-R+r(V), R-r(V))$.

Тогда $f * U^{\lambda, \eta}=0$ для любого $\eta \in\{0, \ldots, m(\lambda, U)-1\}$. В частности, $f=0$, если $\mathscr{Z}(\widehat{U}) \cap \mathscr{Z}(\widehat{V})=\varnothing$.

ДоказАТЕЛЬСтво. Пусть $F=f * U^{\lambda, \eta}$. Из (39) и условия на $V$ получаем, что $F * V=0$ и $\left(-i \frac{d}{d t}-\lambda\right)^{\eta+1} F=0$ в $(-R+r(U), R-r(U))$. Поскольку $\lambda \notin \mathscr{Z}(\widehat{V})$, то $F=0$ (см.,например, [4; часть 3, теорема 1.7]). Более того, если $\mathscr{Z}(\widehat{U}) \cap \mathscr{Z}(\widehat{V})=\varnothing$, то $f=0$ ввиду следствия 3 . 
ДокАЗАТЕЛЬСтво теОРемы 6. Сначала рассмотрим случай $n=1$. Не ограничивая общности, можно считать, что $\mathscr{T}$ состоит из ненулевых распределений и $\mathscr{Z}\left(\widehat{T}_{\alpha}\right) \neq \varnothing$ для любого $\alpha$. Если $r_{*}(\mathscr{T})=r(T)$ при некотором $T \in \mathscr{T}$, то требуемые утверждения очевидны в силу леммы 3 и следствия 2. Предположим теперь, что $r_{*}(\mathscr{T})<r\left(T_{\alpha}\right)$ для всех $\alpha$. Тогда для любого $\varepsilon>0$ множество $A_{\varepsilon}=\left\{\alpha \in A: r\left(T_{\alpha}\right)<r_{*}(\mathscr{T})+\varepsilon\right\}$ непусто. Сначала рассмотрим случай, когда $r^{*}(\mathscr{T})=r(T)$ при некотором $T \in \mathscr{T}$. Пусть $\lambda \in \mathscr{Z}(\widehat{T}), \varepsilon=R-R_{\mathscr{T}}$. Докажем, что

$$
f * T^{\lambda, m(\lambda, T)-1}=0 .
$$

Если существует $\alpha \in A_{\varepsilon}$ такое, что $\lambda \notin \mathscr{Z}\left(\widehat{T}_{\alpha}\right)$, то это следует из леммы 3 при $U=T$ и $V=T_{\alpha}$. В противном случае $\lambda \in \bigcap_{\alpha \in A_{\varepsilon}} \mathscr{Z}\left(\widehat{T}_{\alpha}\right)$. Поскольку $\mathscr{Z}(\mathscr{T})=\varnothing$, существует $\beta \in A$ такое, что $\lambda \notin \mathscr{Z}\left(\widehat{T}_{\beta}\right)$. Пусть $\gamma \in A_{\varepsilon}$ и $\eta=m\left(\lambda, T_{\gamma}\right)-1$. Применяя лемму 3 при $U=T_{\gamma}, V=T_{\beta}$, получаем, что $f * T_{\gamma}^{\lambda, \eta}=0$. Заметим, что $\lambda \notin \mathscr{Z}\left(\widehat{T}_{\gamma}^{\lambda, \eta}\right)$. Используя теперь лемму 3 при $U=T$ и $V=T_{\gamma}^{\lambda, \eta}$, приходим к (53). Утверждение (i) для $n=1$ теперь получаем из следствия 2.

Для доказательства (ii) в одномерном случае выберем произвольное $T \in \mathscr{T}$ и установим (53) для любого $\lambda \in \mathscr{Z}(\widehat{T})$. Пусть $\lambda \in \mathscr{Z}(\widehat{T})$. Если существует $\beta \in A$ такое, что $r\left(T_{\beta}\right) \leqslant r(T)$ и $\lambda \notin \mathscr{Z}\left(\widehat{T}_{\beta}\right)$, то рассуждение выше показывает, что выполнено (53). Остается рассмотреть случай, когда $\lambda \in \mathscr{Z}\left(\widehat{T}_{\alpha}\right)$ для любого $\alpha \in A$ такого, что $r\left(T_{\alpha}\right) \leqslant r(T)$. Так как $\mathscr{Z}(\mathscr{T})=\varnothing$, имеем $\lambda \notin \mathscr{Z}\left(\widehat{T}_{\beta}\right)$ при некотором $\beta \in A$ таком, что $r\left(T_{\beta}\right)>r(T)$. Полагая $\varepsilon=R-r\left(T_{\beta}\right)$, заключаем, что $f * T_{\gamma}^{\lambda, \eta}=0$ при некотором $\gamma \in A_{\varepsilon}, \eta=m\left(\lambda, T_{\gamma}\right)-1$ (см. лемму 3 при $U=T_{\gamma}$, $\left.V=T_{\beta}\right)$. Снова используя лемму 3 при $U=T$ и $V=T_{\gamma}^{\lambda, \eta}$, получаем (53). Из этого результата и следствия 2 получаем (ii). При $n \geqslant 2$ утверждения (i) и (ii) следуют из одномерного случая и теоремы 5.

Как уже отмечалось, для случая, когда существует $\alpha_{0} \in A$ такое, что $T_{\alpha_{0}}$ является гиперболическим, $R>r\left(T_{\alpha_{0}}\right)+r^{*}(\mathscr{T})$ и $\left\{z \in \mathbb{C}^{n}: \widehat{T}_{\alpha}(z)=0 \forall \alpha \in A\right\}=\varnothing$, утверждение (i) теоремы 6 было доказано другими методами К. А. Беренстейном и Р. Гэем [5; теорема 12]. Отметим также, что теорема 6 усиливает второе утверждение в теореме 4.1 из [4; часть 3$]$.

Далее, пусть $M(n)$ - группа движений в $\mathbb{R}^{n}, n \geqslant 2$. Для любых $\psi \in \mathscr{E}^{\prime}\left(\mathbb{R}^{n}\right)$, $g \in M(n)$ определим распределение $g \psi \in \mathscr{E}^{\prime}\left(\mathbb{R}^{n}\right)$ по формуле

$$
\langle g \psi, f(x)\rangle=\left\langle\psi, f\left(g^{-1} x\right)\right\rangle, \quad f \in C^{\infty}\left(\mathbb{R}^{n}\right) .
$$

Если $r(\psi)<R$, положим $M_{\psi, R}=\left\{g \in M(n): \operatorname{supp} g \psi \subset B_{R}\right\}$.

Пусть $\Psi=\left\{\psi_{\alpha}\right\}_{\alpha \in A}$ - семейство распределений из $\mathscr{E}^{\prime}\left(\mathbb{R}^{n}\right)$ такое, что $r\left(\psi_{\alpha}\right)<$ $R \leqslant+\infty$ для всех $\alpha \in A$ (здесь, как и выше, $A$ - некоторое непустое множество индексов). Будем говорить, что $\Psi$ имеет свойство Помпейю в шаре $B_{R}$, если для любой $f \in C^{\infty}\left(B_{R}\right)$ из условия

$$
\left\langle g \psi_{\alpha}, f\right\rangle=0 \quad \text { при всех } \alpha \in A \quad \text { и всех } g \in M_{\psi_{\alpha}, R}
$$

следует, что $f=0$. Наиболее интересный случай (соответствующий классической проблеме Помпейю) возникает, когда семейство $\Psi$ состоит из единственного распределения $\psi$, совпадающего с характеристической функцией некоторого компакта $E$ в $\mathbb{R}^{n}$. В этом случае $E$ называется множеством Помпейю в шаре $B_{R}$, если соответствующее $\Psi$ имеет свойство Помпейю в $B_{R}$. 
Одним из важнейших приложений теоремы 6 является следующий результат.

Tеорема 7. Пусть $n \geqslant 2 u \Psi=\left\{\psi_{\alpha}\right\}_{\alpha \in A}$ - семейство распределений из $\mathscr{E}^{\prime}\left(\mathbb{R}^{n}\right)$ mакое, что $R_{\Psi}<R$ для некоторого $R>0$.

Тогда $\Psi$ имеет свойство Помпейю в шаре $B_{R}$ тогда и только тогда, когда оно имеет свойство Помпейю в $\mathbb{R}^{n}$.

ДокАЗАТЕЛЬство. Пусть $\Psi$ имеет свойство Помпейю в $\mathbb{R}^{n}$, и пусть $\varepsilon=$ $R-\inf _{\alpha} r\left(\psi_{\alpha}\right)-\sup _{\alpha} r\left(\psi_{\alpha}\right)$. Без ограничения общности можно считать, что $\operatorname{supp} \psi_{\alpha} \subset \overline{B_{r\left(\psi_{\alpha}\right)}}$. Положим

$$
\mathscr{A}_{\alpha, \varepsilon}=\left\{h \in M(n): h \overline{B_{r\left(\psi_{\alpha}\right)}} \subset B_{r\left(\psi_{\alpha}\right)+\varepsilon / 3}\right\} .
$$

Для $h \in \mathscr{A}_{\alpha, \varepsilon}$ определим $T_{\alpha, h} \in \mathscr{E}_{\natural}^{\prime}\left(\mathbb{R}^{n}\right)$ равенством $T_{\alpha, h}=\left(h \psi_{j}\right)^{0,1}$ (см. (6)). Заметим, что $\operatorname{supp} T_{\alpha, h} \subset B_{r\left(\psi_{\alpha}\right)+\varepsilon / 3}$. Предположим, что существует $\lambda \in \mathbb{C}$ такое, что $\widetilde{T_{\alpha, h}}(\lambda)=0$ для всех $\alpha$ и всех $h \in \mathscr{A}_{\alpha, \varepsilon}$. Тогда функция $u=\Phi_{\lambda, 0,0,1}$ удовлетворяет уравнению $u * T_{\alpha, h}=0$ при всех $\alpha$ и всех $h \in \mathscr{A}_{\alpha, \varepsilon}$ (см. (16)). Так как $u$ является вещественно-аналитической, то $u *\left(g \psi_{\alpha}\right)^{0,1}=0$ для всех $\alpha$ и всех $g \in M(n)$. Учитывая, что $u$ является радиальной, получаем, что

$$
\left\langle g \psi_{\alpha}, u\right\rangle=\left\langle\left(g \psi_{\alpha}\right)^{0,1}, u\right\rangle=0
$$

для всех $g \in M(n), \alpha \in A$. Это противоречит условию на $\Psi$. Таким образом, $\bigcap_{\alpha} \bigcap_{h \in \mathscr{A}_{\alpha, \varepsilon}} \mathscr{Z}\left(\widehat{T_{\alpha, h}}\right)=\varnothing$. Предположим теперь, что $f \in C^{\infty}\left(B_{R}\right)$ и выполнено (54). Тогда $f * T_{\alpha, h}=0$ для всех $\alpha$ и всех $h \in \mathscr{A}_{\alpha, \varepsilon}$. Из теоремы 6 следует, что $\Psi$ имеет свойство Помпейю в $B_{R}$. Обратное утверждение тривиально.

Из теоремы 7 непосредственно получаем следующий результат.

СлеДСтвиЕ 3. Пусть $E$ - непустое компактное множество в $\mathbb{R}^{n}, n \geqslant 2$, u пусть $r_{E}$ - радиус наименьшего замкнутого шара в $\mathbb{R}^{n}$, содержащего $E$.

Тогда если Е является множеством Помпейю в $\mathbb{R}^{n} u R>2 r_{E}$, то $E$ является множеством Помпейю в шаре $B_{R}$.

Отметим, что для любого $\varepsilon>0$ из неравенства $R>(2-\varepsilon) r_{E}$ не следует, вообще говоря, что $E$ - множество Помпейю в $B_{R}$ (см. [4; часть 4 , теорема 1.6]). Аналоги следствия 3 при различных дополнительных ограничениях, связанных с регулярностью границы множества $E$, были получены в [13] и [4; часть 4].

\section{§ 5. Функции $\phi_{\lambda, \eta, p, q, l}$}

Пусть ${ }_{1} F_{1}(a ; b ; \zeta)(a, \zeta \in \mathbb{C}, b \in \mathbb{C} \backslash\{0,-1,-2, \ldots\})$ - вырожденная гипергеометрическая функция Куммера [17; гл.6]. Для $\lambda \in \mathbb{C}, \eta, p, q \in \mathbb{Z}_{+}$, $l \in\{1, \ldots, d(n, p, q)\}$ и $z=\varrho \sigma \in \mathbb{C}^{n} \backslash\{0\}$ определим

$$
\phi_{\lambda, \eta, p, q, l}(z)=\sqrt{\omega_{2 n-1}} \phi_{\lambda, \eta, p, q}(\varrho) S_{l}^{p, q}(\sigma),
$$

где

$$
\phi_{\lambda, \eta, p, q}(\varrho)=\left.\left(\frac{d}{d \zeta}\right)^{\varkappa}\left(\varrho^{p+q} e_{1}^{-\varrho^{2} / 4} F_{1}\left(p+\frac{n-\zeta^{2}}{2} ; n+p+q ; \frac{\varrho^{2}}{2}\right)\right)\right|_{\zeta=\lambda},
$$


причем $\varkappa=\eta$, если $\lambda \neq 0$, и $\varkappa=2 \eta$, если $\lambda=0$. Легко видеть, что $\phi_{\lambda, \eta, p, q, l}$ допускает непрерывное продолжение в точку нуль. Далее считаем, что $\phi_{\lambda, \eta, p, q, l}$ определена в нуле по непрерывности. Тогда $\phi_{\lambda, \eta, p, q, l} \in \mathrm{RA}\left(\mathbb{C}^{n}\right)$.

Для любого $s \in \mathbb{Z}$ введем дифференциальные операторы $D_{i}(s), i=1,2$, действующие следующим образом:

$$
\left(D_{i}(s) \varphi\right)(\varrho)=\varrho^{s} e^{(-1)^{(i+1)} \varrho^{2} / 4} \frac{d}{d \varrho}\left(\varrho^{-s} e^{(-1)^{i} \varrho^{2} / 4} \varphi(\varrho)\right), \quad \varphi \in C^{1}(0,+\infty) .
$$

ПРЕДЛОЖЕНИЕ 2. При $s=p+q$ справедливъ следующие соотношения:

$$
\begin{array}{rlrl}
D_{1}(s) \phi_{\lambda, 0, p, q} & =\frac{\left(n+2 q+\lambda^{2}\right)}{-2(n+s)} \phi_{\lambda, 0, p, q+1}, & \\
D_{2}(s) \phi_{\lambda, 0, p, q} & =\frac{\left(n+2 p-\lambda^{2}\right)}{2(n+s)} \phi_{\lambda, 0, p+1, q}, & & \\
D_{1}(2-s-2 n) \phi_{\lambda, 0, p, q} & =2(n+s-1) \phi_{\lambda, 0, p-1, q}, & & \\
D_{2}(2-s-2 n) \phi_{\lambda, 0, p, q} & =2(n+s-1) \phi_{\lambda, 0, p, q-1}, & & p \in \mathbb{Z}, \quad q \in \mathbb{Z}_{+} ;
\end{array}
$$

ДокАЗАТЕЛЬСтво. Применим формулу дифференцирования для функции ${ }_{1} F_{1}$ (см. [17; п. 6.4, формула (8)]. Учитывая связь между смежными вырожденными гипергеометрическими функциями (см. [17; п. 6.4, формула (4) и п. 6.4, формула (5)]), приходим к (57)-(59).

ПРЕДЛОЖЕНИЕ 3. Имеет место равенство

$$
\left(\mathfrak{L}-\lambda^{2} \mathrm{Id}\right) \phi_{\lambda, 0, p, q, l}=0 .
$$

ДокАЗАтЕльство. В силу (22) уравнение

$$
\left(\mathfrak{L}-\lambda^{2} \mathrm{Id}\right)\left(f(\varrho) S_{l}^{p, q}(\sigma)\right)=0
$$

можно переписать в виде

$$
f^{\prime \prime}(\varrho)+\frac{f^{\prime}(\varrho)}{\varrho}(2 n-1)-\frac{f(\varrho)}{\varrho^{2}}\left((p+q)(2 n+p+q-2)+\left(p-q-\lambda^{2}\right) \varrho^{2}+\frac{1}{4} \varrho^{4}\right)=0 .
$$

Делая замену $f(\varrho)=\varrho^{p+q} e^{-\varrho^{2} / 4} u\left(\varrho^{2} / 2\right)$, приходим к вырожденному гипергеометрическому уравнению (см. [17; п. 6.1, формула (2)]). Это дает (60).

Напомним теперь разложение Трикоми ${ }_{1} F_{1}(a ; b ; \zeta)$ в ряд по функциям Бесселя (см. [17; п. 6.12, формула (11)]). Положим

$$
A_{k}(\mu, \lambda)=\left.\frac{1}{k !}\left(\frac{d}{d \zeta}\right)^{k}\left(e^{2 \mu \zeta}(1-\zeta)^{\mu-\lambda}(1+\zeta)^{-\mu-\lambda}\right)\right|_{\zeta=0}, \quad k \in \mathbb{Z}_{+}
$$

Коэффициенты $A_{k}(\mu, \lambda)$ обладают следующими свойствами:

1) $A_{0}(\mu, \lambda)=1, A_{1}(\mu, \lambda)=0, A_{2}(\mu, \lambda)=\lambda$,

$$
(k+1) A_{k+1}(\mu, \lambda)=(k+2 \lambda-1) A_{k-1}(\mu, \lambda)-2 \mu A_{k-2}(\mu, \lambda) \quad \text { при } k \geqslant 2 ;
$$


2) если $a_{m, k}(\lambda)=\left.\frac{1}{m !}\left(\frac{d}{d \mu}\right)^{m}\left(A_{k}(\mu, \lambda)\right)\right|_{\mu=0}$, то

$$
A_{k}(\mu, \lambda)=\sum_{m=0}^{[k / 3]} a_{m, k}(\lambda) \mu^{m}
$$

3) $A_{k}(-\mu, \lambda)=(-1)^{k} A_{k}(\mu, \lambda)$.

Формула Трикоми имеет вид

$$
e^{-\zeta / 2}{ }_{1} F_{1}(a ; b ; \zeta)=\Gamma(b)(c \zeta)^{(1-b) / 2} \sum_{k=0}^{\infty} A_{k}\left(c, \frac{b}{2}\right)\left(\frac{\zeta}{4 c}\right)^{k / 2} J_{k+b-1}(2 \sqrt{c \zeta}),
$$

где

$$
c=\frac{b}{2}-a
$$

Мы используем $(63)$ для представления ${ }_{1} F_{1}(a ; b ; \zeta)$ в виде одномерного преобразования Фурье.

ПреДЛОЖЕНИЕ 4. Пусть $\operatorname{Re} b>1 / 2 u x>0$. Тогдa

$$
x^{b-1} e^{-x / 2}{ }_{1} F_{1}(a ; b ; x)=\int_{0}^{2 \sqrt{x}} \cos (\sqrt{c} t) k_{b}(x, t) d t,
$$

где с определяется равенством (64) и

$$
\begin{aligned}
k_{b}(x, t)= & \frac{\Gamma(b)}{\sqrt{\pi} 2^{2 b-3}} \\
& \quad \times \sum_{k=0}^{\infty} \frac{1}{\Gamma(b+k-1 / 2) 8^{k}} \sum_{m=0}^{[k / 3]}(-1)^{m} a_{m, k}\left(\frac{b}{2}\right) \frac{d^{2 m}}{d t^{2 m}}\left(\left(4 x-t^{2}\right)^{k-3 / 2+b}\right) .
\end{aligned}
$$

При этом $k_{b}(x, t)\left(4 x-t^{2}\right)^{-b+3 / 2}$ является бесконечно дифференцируемой функиией переменных $x, t$ на $\mathbb{R}^{2}$.

ДокАзАТЕЛЬство. Нетрудно видеть, что

$$
\frac{d^{2 m}}{d t^{2 m}}\left(\left(4 x-t^{2}\right)^{k-3 / 2+b}\right)=\sum_{j=0}^{m} c_{j, m, k}(b) t^{2 j}\left(4 x-t^{2}\right)^{k-3 / 2+b-m-j},
$$

где

$$
\left|c_{j, m, k}(b)\right| \leqslant(2(k+|b|+3 m+2))^{2 m}, \quad 0 \leqslant j \leqslant m .
$$

Кроме того, используя индукцию по $k$, из (61) и (62) легко получить неравенстBO

$$
\left|a_{m, k}\left(\frac{b}{2}\right)\right| \leqslant(|b|+2)^{k}, \quad 0 \leqslant m \leqslant\left[\frac{k}{3}\right] .
$$

Оценки (67) и (68) показывают, что функция (66) определена корректно и

$$
k_{b}(x, t)\left(4 x-t^{2}\right)^{-b+3 / 2} \in C^{\infty}\left(\mathbb{R}^{2}\right) .
$$


Далее, ввиду (63) и представления Пуассона для функций Бесселя (см. [17; п. 7.12, формула (8)]),

$$
\begin{aligned}
x^{b-1} e^{-x / 2}{ }_{1} F_{1}(a ; b ; x) & \\
& =\frac{\Gamma(b)}{\sqrt{\pi} 2^{2 b-3}} \sum_{k=0}^{\infty} \frac{A_{k}(c, b / 2)}{\Gamma(b+k-1 / 2)} \int_{0}^{2 \sqrt{x}} \cos (\sqrt{c} t)\left(4 x-t^{2}\right)^{k-3 / 2+b} d t .
\end{aligned}
$$

Повторное интегрирование по частям дает

$$
\begin{aligned}
& c^{j} \int_{0}^{2 \sqrt{x}} \cos (\sqrt{c} t)\left(4 x-t^{2}\right)^{k-3 / 2+b} d t \\
& \quad=(-1)^{j} \int_{0}^{2 \sqrt{x}} \cos (\sqrt{c} t) \frac{d^{2 j}}{d t^{2 j}}\left(\left(4 x-t^{2}\right)^{k-3 / 2+b}\right) d t
\end{aligned}
$$

при $0 \leqslant j \leqslant k / 2$. Отсюда (см. (62))

$$
\begin{aligned}
& A_{k}\left(c, \frac{b}{2}\right) \int_{0}^{2 \sqrt{x}} \frac{\cos (\sqrt{c} t)}{\left(4 x-t^{2}\right)^{3 / 2-k-b}} d t \\
& \quad=\int_{0}^{2 \sqrt{x}} \cos (\sqrt{c} t) \sum_{m=0}^{[k / 3]}(-1)^{m} a_{m, k}\left(\frac{b}{2}\right) \frac{d^{2 m}}{d t^{2 m}}\left(\left(4 x-t^{2}\right)^{k-3 / 2+b}\right) d t .
\end{aligned}
$$

Подставляя (70) в (69) и принимая во внимание (67) и (68), получаем интегральное представление (65).

СледСтвиЕ 4. Справедливо интегральное представление

$$
\phi_{\lambda, 0, p, q}(\varrho)=\frac{1}{\sqrt{\omega_{2 n-1}}} \int_{0}^{\varrho} \cos (\lambda t) \mathfrak{K}_{n, p, q}(\varrho, t) d t,
$$

где $\mathfrak{K}_{n, p, q}(\varrho, \cdot) \in L^{1}[0, \varrho]$. При этом

$$
\begin{aligned}
\mathfrak{K}_{n, p, p}(\varrho, t)=4^{n+2 p-1} \sqrt{\omega_{2 n-1}} \frac{\left(\varrho^{2}-t^{2}\right)^{n+2 p-3 / 2}}{\varrho^{2 n+2 p-2}} \\
\quad \times\left.\left(k_{n+2 p}(u, v)\left(4 u-v^{2}\right)^{3 / 2-n-2 p}\right)\right|_{u=\varrho^{2} / 2, v=\sqrt{2} t},
\end{aligned}
$$

где $k_{n+2 p}(u, v)$ определяется равенством (66).

ДокАЗАТЕльство. Согласно (56) и предложению 4

$$
\phi_{\lambda, 0, p, q}(\varrho)=\frac{1}{\varrho^{n+b-2}} \int_{0}^{\varrho} \cos \left(\sqrt{\lambda^{2}+q-p} t\right)\left(\varrho^{2}-t^{2}\right)^{n+p+q-3 / 2} \mathscr{K}_{n, p, q}(\varrho, t) d t,
$$

где

$$
\mathscr{K}_{n, p, q}(\varrho, t)=\left.4^{n+p+q-1} k_{n+p+q}(u, v)\left(4 u-v^{2}\right)^{3 / 2-n-p-q)}\right|_{u=\varrho^{2} / 2, v=\sqrt{2} t} .
$$

Используя (72), асимптотику для ${ }_{1} F_{1}$ (см. [17; п. 6.13, формула $\left.(26)\right]$ ) и теорему Винера-Пэли, мы получаем (71). 
ПреДЛОЖеНИЕ 5. Пусть $0<r<\infty, \alpha, \beta \in \mathbb{Z}_{+}^{n}$. Тогда при $\lambda \rightarrow \infty$ имеет место оиенка

$$
\sup _{z \in \mathscr{B}_{r}}\left|\frac{\partial^{|\alpha|+|\beta|}}{\partial z^{\alpha} \partial \bar{z}^{\beta}} \phi_{\lambda, \eta, p, q, l}(z)\right|=O\left(|\lambda|^{|\alpha|+|\beta|-p-q} e^{r|\operatorname{Im} \lambda|}\right) .
$$

ДокАЗАТЕЛЬСтво. Положим

$$
f(t)=e^{-t / 4}{ }_{1} F_{1}\left(p+\frac{n-\lambda^{2}}{2} ; n+p+q ; \frac{t}{2}\right) .
$$

Индукцией по $|\alpha|+|\beta|$ легко доказать, что

$$
\frac{\partial^{|\alpha|+|\beta|}}{\partial z^{\alpha} \partial \bar{z}^{\beta}} \phi_{\lambda, 0, p, q, l}(z)=\sum_{k \geqslant \max \{0, m / 2\}}^{|\alpha|+|\beta|} f^{(k)}\left(|z|^{2}\right) \mathrm{Q}_{2 k-m}(z),
$$

где $m=|\alpha|+|\beta|-p-q$ и $\mathrm{Q}_{2 k-m}$ является однородным многочленом в $\mathbb{C}^{n}$ степени $2 k-m$. В силу формулы дифференцирования для ${ }_{1} F_{1}$ (см. [17; п. 6.4, формула (8)]) мы можем записать (74) в виде

$$
\begin{gathered}
\frac{\partial^{|\alpha|+|\beta|}}{\partial z^{\alpha} \partial \bar{z}^{\beta}} \phi_{\lambda, 0, p, q, l}(z)=\sum_{k \geqslant \max \{0, m / 2\}}^{|\alpha|+|\beta|} \sum_{j=0}^{k}(-1)^{k-j}\left(\begin{array}{l}
j \\
k
\end{array}\right) \frac{2^{j}}{4^{k}} \frac{\left(p+\left(n-\lambda^{2}\right) / 2\right)_{j}}{(n+p+q)_{j}} \\
\times{ }_{1} F_{1}\left(p+j+\frac{n-\lambda^{2}}{2} ; n+p+q+j ; \frac{|z|^{2}}{2}\right) \mathrm{Q}_{2 k-m}(z) e^{-|z|^{2} / 4}
\end{gathered}
$$

Предположим, что $|\lambda|^{-1} \leqslant|z| \leqslant r$. Тогда из $(75)$ и асимптотики для ${ }_{1} F_{1}$ (см. [17; п. 6.13 , формула (26)]) имеем

$$
\frac{\partial^{|\alpha|+|\beta|}}{\partial z^{\alpha} \partial \bar{z}^{\beta}} \phi_{\lambda, 0, p, q, l}(z)=O\left(|\lambda|^{|\alpha|+|\beta|-p-q} e^{r|\operatorname{Im} \lambda|}\right) .
$$

Аналогично, при $|z| \leqslant|\lambda|^{-1}$ соотношение (75) и [17; п. 6.13, формула (15)] дают

$$
\frac{\partial^{|\alpha|+|\beta|}}{\partial z^{\alpha} \partial \bar{z}^{\beta}} \phi_{\lambda, 0, p, q, l}(z)=O\left(|\lambda|^{|\alpha|+|\beta|-p-q}\right) .
$$

Комбинируя (76) и (77), получаем (73) для $\eta=0$. Отсюда и из интегральной формулы Коши следует (73) в общем случае.

$$
\begin{aligned}
& \text { Для } j \in \mathbb{Z}_{+} \text {положим } \\
& \lambda_{j}=\sqrt{2 p+n+2 j}, \quad \mu_{j}=\frac{2^{1-n-p-q}}{\omega_{2 n-1}(n+p+q-1) !}\left(\begin{array}{c}
n+p+q+j-1 \\
n+p+q-1
\end{array}\right) .
\end{aligned}
$$

В терминах многочленов Лагерра $L_{j}^{\alpha}$ (см. $[17 ;$ п. 6.9 , формула $\left.(36)]\right)$ имеем

$$
\left(\begin{array}{c}
n+p+q+j-1 \\
n+p+q-1
\end{array}\right) \phi_{\lambda_{j}, 0, p, q}(\varrho)=\varrho^{p+q} e^{-\varrho^{2} / 4} L_{j}^{n+p+q-1}\left(\frac{\varrho^{2}}{2}\right)
$$


ПРеДЛОженИЕ 6. Система функиий $\left\{\phi_{\lambda_{j}, 0, p, q, l}\right\}_{j=0}^{\infty}$ образует ортогональный базис в $L_{p, q, l}^{2}\left(\mathbb{C}^{n}\right)$. Кроме того,

$$
\int_{\mathbb{C}^{n}}\left|\phi_{\lambda_{j}, 0, p, q, l}(z)\right|^{2} d m_{n}(z)=\frac{1}{\mu_{j}}
$$

где $d m_{n}(z)$ - мера Лебега на $\mathbb{C}^{n}$.

ДокАЗАТЕЛЬСтво. Пусть

$$
g \in L^{2}\left((0,+\infty), t^{n+p+q-1} e^{-t} d t\right), \quad G(z)=\varrho^{p+q} e^{-\varrho^{2} / 4} g\left(\frac{\varrho^{2}}{2}\right) S_{l}^{p, q}(\sigma) .
$$

Тогда

$$
\begin{aligned}
\int_{\mathbb{C}^{n}}|G(z)|^{2} d m_{n}(z) & =\int_{0}^{\infty} \int_{\mathbb{S}^{2 n-1}} \varrho^{2(n+p+q)-1} e^{-\varrho^{2} / 2}\left|g\left(\frac{\varrho^{2}}{2}\right)\right|^{2}\left|S_{l}^{p, q}(\sigma)\right|^{2} d \varrho d \omega(\sigma) \\
& =2^{n+p+q-1} \int_{0}^{\infty} t^{n+p+q-1} e^{-t}|g(t)|^{2} d t
\end{aligned}
$$

Поэтому отображение $g \rightarrow G$ является изоморфизмом пространства $L^{2}((0,+\infty)$, $\left.(2 t)^{n+p+q-1} e^{-t} d t\right)$ на пространство $L_{p, q, l}^{2}\left(\mathbb{C}^{n}\right)$. Это завершает доказательство, поскольку многочлены $L_{j}^{\alpha}, j \in \mathbb{Z}_{+}$, образуют ортогональный базис в пространстве $L^{2}\left((0,+\infty), t^{\alpha} e^{-t} d t\right)$ и

$$
\int_{0}^{\infty} t^{\alpha} e^{-t}\left(L_{j}^{\alpha}(t)\right)^{2} d t=\frac{\Gamma(\alpha+j+1)}{j !}
$$

(см. [17; п. 10.12, формула (2)]).

\section{§ 6. Аналоги сферического преобразования}

Пусть $p, q \in \mathbb{Z}_{+}, l \in\{1, \ldots, d(n, p, q)\}$. Для любого $f \in \mathscr{E}_{p, q, l}^{\prime}\left(\mathbb{C}^{n}\right)$ положим

$$
\mathscr{F}_{l}^{p, q}(f)(\lambda)=\left\langle f, \overline{\phi_{\bar{\lambda}, 0, p, q, l}}\right\rangle=\sqrt{\omega_{2 n-1}}\left\langle f, \phi_{\lambda, 0, p, q}(\varrho) \overline{S_{l}^{p, q}(\sigma)}\right\rangle, \quad \lambda \in \mathbb{C} .
$$

Согласно $(55) \mathscr{F}_{l}^{p, q}(f)$ является четной целой функцией по $\lambda$. Если $f \in \mathscr{E}_{\natural}^{\prime}\left(\mathbb{C}^{n}\right)$, то мы во многих случаях будем писать $\widetilde{f}(\lambda)$ вместо $\mathscr{F}_{1}^{0,0}(f)(\lambda)$, т.е. $\tilde{f}(\lambda)=$ $\left\langle f, \phi_{\lambda, 0,0,0,1}\right\rangle$. Изучим основные свойства преобразования $\mathscr{F}_{l}^{p, q}$.

ПреДЛОЖениЕ 7. Пусть $T \in \mathscr{E}_{\sharp}^{\prime}\left(\mathbb{C}^{n}\right)$. Предположим, ито $R \in(r(T),+\infty]$, $f \in \mathscr{D}^{\prime}\left(\mathscr{B}_{R}\right)$ u $\mathfrak{L} f=\lambda^{2} f$ при некотором $\lambda \in \mathbb{C}$. Тогда в шаре $\mathscr{B}_{R-r(T)}$

$$
f \times T=\widetilde{T}(\lambda) f
$$

ДокАзАТЕльство. Так как $\mathfrak{L}$ является эллиптическим оператором, распределение $f$ принадлежит $\operatorname{RA}\left(\mathscr{B}_{R}\right)$ (см. [6; гл. 8.6]). Зафиксируем $w \in \mathscr{B}_{R-r(T)}$ и введем функцию (см. §1)

$$
f_{w}(z)=\int_{U(n)}(\tau(w) f)(U z) d U, \quad z \in \mathscr{B}_{R-|w|}
$$


где $d U$ - нормированная мера Хаара на группе $U(n)$. Из определения $f_{w}$ видно, что

$$
f_{w} \in \mathrm{RA}_{\natural}\left(\mathscr{B}_{R-|w|}\right), \quad f_{w}(0)=f(w) .
$$

Кроме того, ввиду условия на $f$ и $(21)$

$$
\left(\mathfrak{L} f_{w}\right)(z)=\lambda^{2} f_{w}(z), \quad z \in \mathscr{B}_{R-|w|} .
$$

Используя (81), (82) и (22), получаем, что $f_{w}(z)=f(w) \phi_{\lambda, 0,0,0,1}(z)$. Поскольку $T \in \mathscr{E}_{\sharp}^{\prime}\left(\mathbb{C}^{n}\right)$, то

$$
\widetilde{T}(\lambda) f(w)=\left\langle T(z), f_{w}(z)\right\rangle=\langle T(z), \tau(w) f(z)\rangle=(f \times T)(w),
$$

что и требовалось.

ПреДЛОжениЕ 8. Если $f \in \mathscr{E}_{p, q, l}^{\prime}\left(\mathbb{C}^{n}\right) u T \in \mathscr{E}_{\natural}^{\prime}\left(\mathbb{C}^{n}\right)$, mo

$$
\mathscr{F}_{l}^{p, q}(f \times T)(\lambda)=\mathscr{F}_{l}^{p, q}(f)(\lambda) \widetilde{T}(\lambda) .
$$

ДокАЗАТЕЛЬСтво. Из (60) и (80) имеем

$$
\left\langle f \times T, \overline{\phi_{\bar{\lambda}, 0, p, q, l}}\right\rangle=\left\langle f, \overline{\phi_{\bar{\lambda}, 0, p, q, l} \times \bar{T}}\right\rangle=\left\langle f, \overline{\widetilde{T}(\bar{\lambda})} \overline{\phi_{\bar{\lambda}, 0, p, q, l}}\right\rangle=\widetilde{T}(\lambda)\left\langle f, \overline{\phi_{\bar{\lambda}, 0, p, q, l}}\right\rangle,
$$

что доказывает (83) (см. (79) и (23)).

ПреДЛОЖЕНИЕ 9. Преобразование $\mathscr{F}_{l}^{p, q}$ инбективно на $\mathscr{E}_{p, q, l}^{\prime}\left(\mathbb{C}^{n}\right)$.

ДокАЗАтельСтво. Пусть $f \in \mathscr{E}_{p, q, l}^{\prime}\left(\mathbb{C}^{n}\right)$ и $\mathscr{F}_{l}^{p, q}(f)=0$. В соответствии с (83)

$$
\mathscr{F}_{l}^{p, q}(f \times \varphi)(\lambda)=0, \quad \lambda \in \mathbb{C},
$$

для любой функции $\varphi \in \mathscr{D}_{\sharp}\left(\mathbb{C}^{n}\right)$. Соотношения $(84)$ и $(72)$ влекут

$$
\begin{aligned}
\int_{0}^{a} \cos ( & \left.\sqrt{\lambda^{2}+q-p} x\right) \int_{x}^{a} \varrho^{1-p-q}(f \times \varphi)_{p, q, l}(\varrho) \\
& \times\left(\varrho^{2}-x^{2}\right)^{n+p+q-3 / 2} \mathscr{K}_{n, p, q}(\varrho, x) d \varrho d x=0,
\end{aligned}
$$

где $a=r(f \times \varphi)$. Отсюда

$$
\int_{x}^{a} \varrho^{1-p-q}(f \times \varphi)_{p, q, l}(\varrho)\left(\varrho^{2}-x^{2}\right)^{n+p+q-3 / 2} \mathscr{K}_{n, p, q}(\varrho, x) d \varrho=0
$$

при $0<x<a$. Используя стандартный метод решения интегрального уравнения Абеля (см., например, [20; гл. 1, доказательство теоремы 2.6]), получаем

$$
\frac{(f \times \varphi)_{p, q, l}(\sqrt{t})}{(\sqrt{t})^{p+q}}-\int_{t}^{a^{2}} \frac{(f \times \varphi)_{p, q, l}(\sqrt{s})}{(\sqrt{s})^{p+q}} k(s, t) d s=0,
$$

где

$$
\begin{aligned}
& k(s, t)=\frac{2^{n+p+q-2}}{(2 n+2 p+2 q-2) !}\left(\frac{\partial}{\partial t}\right)^{2 n+2 p+2 q-1}\left((s-t)^{2 n+2 p+2 q-2} g(s, t)\right), \\
& g(s, t)=\int_{-1}^{1}\left(1-x^{2}\right)^{n+p+q-3 / 2} \mathscr{K}_{n, p, q}\left(\sqrt{s}, \sqrt{\frac{s+t-(s-t) x}{2}}\right) d x .
\end{aligned}
$$


Так как $g(s, t) \in C^{\infty}\left(\mathbb{R}^{2}\right)$, то функция $(\sqrt{t})^{-p-q}(f \times \varphi)_{p, q, l}(\sqrt{t})$ является решением однородного интегрального уравнения Вольтерра второго рода с ядром $k \in C^{\infty}\left(\mathbb{R}^{2}\right)$. Поэтому $(f \times \varphi)_{p, q, l}=0$ и $f \times \varphi=0$. В силу произвольности $\varphi$ заключаем, что $f=0$.

ПреДЛОЖеНИЕ 10. (i) Пусть $f \in\left(C^{s} \cap \mathscr{E}_{p, q, l}^{\prime}\right)\left(\mathbb{C}^{n}\right)$ при некотором $s \in \mathbb{Z}_{+}$. Тогда

$$
\left|\mathscr{F}_{l}^{p, q}(f)(\lambda)\right| \leqslant c \frac{e^{r(f)|\operatorname{Im} \lambda|}}{(1+|\lambda|)^{s+p+q}}, \quad \lambda \in \mathbb{C},
$$

где константа с не зависит от $\lambda$.

(ii) Пусть $f \in \mathscr{E}_{p, q, l}^{\prime}\left(\mathbb{C}^{n}\right), s \in \mathbb{Z}_{+}$. Предположим, что

$$
\mathscr{F}_{l}^{p, q}(f)\left(\lambda_{j}\right)=O\left(j^{-n-(p+q+s) / 2-1}\right), \quad j \rightarrow+\infty,
$$

где $\lambda_{j}$ определяется равенством (78). Тогда $f \in C^{s}\left(\mathbb{C}^{n}\right)$.

ДокАзАТЕЛЬство. (i) Согласно (79) имеем

$$
\mathscr{F}_{l}^{p, q}(f)(\lambda)=\sqrt{\omega_{2 n-1}} \int_{0}^{\infty} \varrho^{2 n-1} f_{p, q, l}(\varrho) \phi_{\lambda, 0, p, q}(\varrho) d \varrho .
$$

Для функции $\phi_{\lambda, 0, p, q}$ мы имеем формулы дифференцирования (57)-(59). Поэтому повторное интегрирование по частям в (85) дает

$$
\mathscr{F}_{l}^{p, q}(f)(\lambda)=\kappa \int_{0}^{r(f)} \varrho^{2 n-1}\left(d_{2}^{s-2[s / 2]}\left(d_{1} d_{2}\right)^{[s / 2]} f_{p, q, l}\right)(\varrho) \phi_{\lambda, 0, p+s-2[s / 2], q}(\varrho) d \varrho,
$$

где

$$
\begin{gathered}
d_{1}=D_{1}(1-2 n-p-q), \quad d_{2}=D_{2}(p+q), \\
\kappa=\sqrt{\omega_{2 n-1}}\left(2 p+n-\lambda^{2}\right)^{-[s / 2]}(-2(n+p+q))^{-s+2[s / 2]} .
\end{gathered}
$$

Теперь (i) следует из (73). Утверждение (ii) доказывается непосредственно с помощью предложения $6,(79)$ и (73).

Для $r \in[0,+\infty)$ положим $\overline{\mathscr{B}_{r}}=\left\{z \in \mathbb{C}^{n}:|z| \leqslant r\right\}$. Следующее утверждение является аналогом теоремы Винера-Пэли для $\mathscr{F}_{l}^{p, q}$.

TeOpema 8. (i) Пусть $f \in \mathscr{E}_{p, q, l}^{\prime}\left(\mathbb{C}^{n}\right) u \operatorname{supp} f \subset \overline{\mathscr{B}_{r}}$. Тогда

$$
\left|\mathscr{F}_{l}^{p, q}(f)(\lambda)\right| \leqslant c_{1}(1+|\lambda|)^{c_{2}} e^{r|\operatorname{Im} \lambda|} \quad \text { для всех } \lambda \in \mathbb{C},
$$

где $c_{1}, c_{2}>0$ не зависят от $\lambda$. Обратно, для каждой четной целой функции $w(\lambda)$, удовлетворяющей оченке вида (86), существует такое распределение $f \in \mathscr{E}_{p, q, l}^{\prime}\left(\mathbb{C}^{n}\right)$, чmo

$$
\operatorname{supp} f \subset \overline{\mathscr{B}_{r}}, \quad \mathscr{F}_{l}^{p, q}(f)=w .
$$

(ii) Eсли $f \in \mathscr{D}_{p, q, l}\left(\mathbb{C}^{n}\right)$ u $\operatorname{supp} f \subset \overline{\mathscr{B}_{r}}$, то для любого $N \in \mathbb{Z}_{+}$существует константа $c_{N}>0$ такая, что

$$
\left|\mathscr{F}_{l}^{p, q}(f)(\lambda)\right| \leqslant c_{N}(1+|\lambda|)^{-N} e^{r|\operatorname{Im} \lambda|}, \quad \lambda \in \mathbb{C} .
$$

Обратно, для каждой четной целой функции $w(\lambda)$, удовлетворяющей оченке вида (88) для всех $N \in \mathbb{Z}_{+}$, существует функиия $f \in \mathscr{D}_{p, q, l}\left(\mathbb{C}^{n}\right)$ такая, что выполнены условия (87). 
ДокАЗАТЕльство. (i) Используя (73), получаем для любого $\varepsilon>0$ оценку

$$
\left|\mathscr{F}_{l}^{p, q}(f)(\lambda)\right| \leqslant \kappa_{\varepsilon}(1+|\lambda|)^{\operatorname{ord}(f)-p-q} e^{(r+\varepsilon)|\operatorname{Im} \lambda|}, \quad \lambda \in \mathbb{C},
$$

где $\operatorname{ord}(f)$ - порядок распределения $f$, а $\kappa_{\varepsilon}>0$ не зависит от $\lambda$. Применяя принцип Фрагмена-Линделёфа (см., например, доказательство леммы 4.3 в [15; гл. 3]), приходим к (86).

Докажем обратное утверждение. Сначала предположим, что число нулей функции $w$ конечно. Тогда по теореме Адамара $w$ является четным многочленом. Рассмотрим дифференциальный оператор $S_{l}^{p, q}(\partial)$, ассоциированный с $S_{l}^{p, q}(z)=\varrho^{p+q} S_{l}^{p, q}(\sigma)$, т.е. каждый моном $z_{1}^{\alpha_{1}} \cdots z_{n}^{\alpha_{n}} \bar{z}_{1}^{\beta_{1}} \cdots \bar{z}_{n}^{\beta_{n}}$ заменяется в $S_{l}^{p, q}(z)$ на

$$
2^{p+q}\left(\frac{\partial}{\partial \bar{z}_{1}}\right)^{\alpha_{1}} \cdots\left(\frac{\partial}{\partial \bar{z}_{n}}\right)^{\alpha_{n}}\left(\frac{\partial}{\partial z_{1}}\right)^{\beta_{1}} \cdots\left(\frac{\partial}{\partial z_{n}}\right)^{\beta_{n}}
$$

Для $g \in C^{p+q}\left(B_{\varepsilon}\right), \varepsilon>0$, имеем

$$
S_{l}^{p, q}(\partial)\left(|z|^{2 N} g(z)\right)(0)=0, \quad N \in \mathbb{N} .
$$

Поэтому

$$
\mathscr{F}_{l}^{p, q}\left(S_{l}^{p, q}(\partial)^{*} \delta_{0}\right)(\lambda)=\frac{2^{p+q}(n+p+q-1) !}{\sqrt{\omega_{2 n-1}}(n-1) !},
$$

где оператор $S_{l}^{p, q}(\partial)^{*}$ является сопряженным к оператору $S_{l}^{p, q}(\partial)$. Комбинируя последнее равенство с предложением 8 , делаем вывод, что условия (87) выполнены для распределения

$$
f=P_{1}(\mathfrak{L}) S_{l}^{p, q}(\partial)^{*} \delta_{0}, \quad \text { где } P_{1}(\lambda)=\frac{\sqrt{\omega_{2 n-1}}(n-1) !}{2^{p+q}(n+p+q-1) !} w(\sqrt{\lambda}) .
$$

Далее, пусть функция $w$ имеет бесконечно много нулей. Выберем четный полином $P_{2}: \mathbb{R} \rightarrow \mathbb{C}$, для которого функция $w / P_{2}$ является целой и

$$
\sup _{\lambda \in \mathbb{C}} \frac{(1+|\lambda|)^{2 n+3}}{e^{r|\operatorname{Im} \lambda|}}\left|\frac{w(\lambda)}{P_{2}(\lambda)}\right|<\infty .
$$

По теореме Винера-Пэли существует четная функция $\varphi \in C^{2 n}\left(\mathbb{R}^{1}\right)$ такая, что $\operatorname{supp} \varphi \subset[-r, r]$ и

$$
\frac{w(\lambda)}{P_{2}(\lambda)}=\int_{0}^{r} \cos (\lambda t) \varphi(t) d t, \quad \lambda \in \mathbb{C} .
$$

Пусть $\psi \in C(0, r]$ и удовлетворяет уравнению

$$
\varphi(t)=\omega_{2 n-1} \int_{t}^{r} \varrho \psi(\varrho)\left(\varrho^{2}-t^{2}\right)^{n-3 / 2} \mathscr{K}_{n, 0,0}(\varrho, t) d \varrho, \quad 0<t<r
$$

(как и выше, это уравнение сводится к интегральному уравнению Вольтерра второго рода). Положим $h(z)=\psi(|z|)$ при $z \in \mathscr{B}_{r}$ и $h(z)=0$, если $z \in \mathbb{C}^{n} \backslash \mathscr{B}_{r}$. Нетрудно видеть, что $h \in L\left(\mathbb{C}^{n}\right)$. Кроме того, в силу $(71)$, (89) и (90) имеем 
$\widetilde{h}(\lambda)=w(\lambda) / P_{2}(\lambda)$. Теперь, как и в первом случае, заключаем, что условия $(87)$ выполнены для распределения

$$
f=P_{3}(\mathfrak{L}) S_{l}^{p, q}(\partial)^{*} \delta_{0} \times h, \quad \text { где } P_{3}(\lambda)=\frac{\sqrt{\omega_{2 n-1}}}{2^{p+q}(n)_{p+q}} P_{2}(\sqrt{\lambda}) .
$$

Таким образом, утверждение (i) доказано. Утверждение (ii) непосредственно следует из (i) и предложения 10.

СлеДСТвиЕ 5. Пусть $r>0, u \in L[0, r] u$

$$
\int_{0}^{\varrho} u(t) \mathfrak{K}_{n, p, q}(\varrho, t) d t=0
$$

для почти всех $\varrho \in(0, r)$, где $\mathfrak{K}_{n, p, q}(\varrho, t)$ определено в (71). Тогда $u=0$.

ДоказАтельство. Пусть $\psi \in \mathscr{D}_{\natural}(-r, r)$. По теореме Винера-Пэли и теореме 8 существует функция $h \in \mathscr{D}_{p, q, 1}\left(\mathbb{C}^{n}\right)$ с носителем в $\mathscr{B}_{r}$ такая, что $\widehat{\psi}=$ $\mathscr{F}_{1}^{p, q}(h)$. Используя $(71)$ и $(79)$, из этого равенства находим

$$
\psi(t)=\frac{1}{2} \int_{t}^{r} \varrho^{2 n-1} h_{p, q, 1}(\varrho) \mathfrak{K}_{n, p, q}(\varrho, t) d \varrho, \quad t \in(0, r) .
$$

Тогда по условию получаем

$$
\int_{0}^{r} u(t) \psi(t) d t=0
$$

Поскольку функция $\psi$ выше выбиралась произвольно, $u$ является нулевой на $[0, r]$.

В заключение приведем формулу обращения для $\mathscr{F}_{l}^{p, q}$.

ПредЛожениЕ 11. Пусть $f \in\left(\mathscr{E}_{p, q, l}^{\prime} \cap C^{s}\right)\left(\mathbb{C}^{n}\right)$ при некотором $s \geqslant 2 n+2$. Тогда

$$
\mu_{j} \mathscr{F}_{l}^{p, q}(f)\left(\lambda_{j}\right)=O\left(j^{(2 n+p+q-2-s) / 2}\right), \quad j \rightarrow+\infty,
$$

u

$$
f(z)=\sum_{j=0}^{\infty} \mu_{j} \mathscr{F}_{l}^{p, q}(f)\left(\lambda_{j}\right) \phi_{\lambda_{j}, 0, p, q, l}(z), \quad z \in \mathbb{C}^{n},
$$

где $\lambda_{j}$ и $\mu_{j}$ определены в (78).

ДокАзАтельство. Оценка (91) следует из предложения 10, (i). Соотношение (92) является частным случаем разложения по многочленам Лагерра (см. предложения 5 и 6 ).

\section{§ 7. Уравнения свертки на редуцированной группе Гейзенберга}

Сначала мы определим оператор $\mathfrak{A}_{p, q, l}$, который является аналогом оператора $\mathfrak{A}_{k, j}$ из $\S 2$ для искаженной свертки на $\mathbb{C}^{n}$.

В силу теоремы 8 и теоремы Винера-Пэли отображение $\Lambda: \mathscr{E}_{\sharp}^{\prime}\left(\mathbb{C}^{n}\right) \rightarrow \mathscr{E}_{\natural}^{\prime}\left(\mathbb{R}^{1}\right)$, заданное равенством

$$
\widehat{\Lambda(T)}(\lambda)=\widetilde{T}(\lambda)=\left\langle T, \phi_{\lambda, 0,0,0,1}\right\rangle, \quad \lambda \in \mathbb{C},
$$

является биекцией и $r(\Lambda(T))=r(T)$. 
Пусть $R \in(0,+\infty], f \in \mathscr{D}_{p, q, l}^{\prime}\left(\mathscr{B}_{R}\right)$. Для $\psi \in \mathscr{D}(-R, R)$ выберем $\eta \in \mathscr{D}_{\sharp}\left(\mathscr{B}_{R}\right)$ так, что $\eta=1$ в $\mathscr{B}_{r_{0}(\psi)+\varepsilon}$ при некотором $\varepsilon \in\left(0, R-r_{0}(\psi)\right)$, где $r_{0}(\psi)=\inf \{r>0$ : $\left.\operatorname{supp} \psi \subset \mathscr{B}_{r}\right\}$. Положим

$$
\left\langle\mathfrak{A}_{p, q, l}(f), \psi\right\rangle=\sum_{j=0}^{\infty} \mu_{j} \mathscr{F}_{l}^{p, q}(f \eta)\left(\lambda_{j}\right) \int_{-R}^{R} \psi(t) \cos \left(\lambda_{j} t\right) d t
$$

(см. (78)). Учитывая (71) и предложения 8 и 11, видим из следствия 5 и доказательства леммы 1 , что $\mathfrak{A}_{p, q, l}(f)$ корректно определено посредством (94) как распределение в $\mathscr{D}_{\sharp}^{\prime}(-R, R)$ и $\mathfrak{A}_{p, q, l}\left(\left.f\right|_{\mathscr{B}_{r}}\right)=\left.\mathfrak{A}_{p, q, l}(f)\right|_{(-r, r)}$ для любого $r \in(0, R]$. Кроме того, используя (73), (60), (80) и повторяя рассуждения в доказательстве теоремы 1 , получаем следующий результат.

Теорема 9. При $R \in(0,+\infty], N \in \mathbb{Z}_{+} u \nu=2+2 n+p+q+N$ имеют место следующие утверждения.

(i) Ecли $f \in \mathscr{D}_{p, q, l}^{\prime}\left(\mathscr{B}_{R}\right), T \in \mathscr{E}_{\natural}^{\prime}\left(\mathbb{C}^{n}\right)$ u $r(T)<R$, то на $(r(T)-R, R-r(T))$ справедливо соотношение $\mathfrak{A}_{p, q, l}(f \times T)=\mathfrak{A}_{p, q, l}(f) * \Lambda(T)$.

(ii) Пусть $f \in \mathscr{D}_{p, q, l}^{\prime}\left(\mathscr{B}_{R}\right), r \in(0, R]$. Тогда $f=0$ на $\mathscr{B}_{r}$ в том и толъко том случае, когда $\mathfrak{A}_{p, q, l}(f)=0$ на $(-r, r)$.

(iii) Eсли $f \in C_{p, q, l}^{\nu}\left(\mathscr{B}_{R}\right)$, то $\mathfrak{A}_{p, q, l}(f) \in C_{\natural}^{N}(-R, R)$. Кроме того,

$$
f_{p, q, l}(\varrho)=\int_{0}^{\varrho} \mathfrak{A}_{p, q, l}(f)(t) \mathfrak{K}_{n, p, q}(\varrho, t) d t, \quad 0<\varrho<R,
$$

где $\mathfrak{K}_{n, p, q}(\varrho, t)$ задается равенством (71).

(iv) Отображение $\mathfrak{A}_{p, q, l}$ непрерьвно из $\mathscr{D}_{p, q, l}^{\prime}\left(\mathscr{B}_{R}\right)$ в $\mathscr{D}_{\natural}^{\prime}(-R, R)$, а также из $C_{p, q, l}^{\nu}\left(\mathscr{B}_{R}\right)$ в $C_{\natural}^{N}(-R, R)$.

(v) Дія $\lambda \in \mathbb{C}, \mu \in \mathbb{Z}_{+}$имеем $\mathfrak{A}_{p, q, l}\left(\phi_{\lambda, \mu, p, q, l}\right)=u_{\lambda, \mu}$, где $u_{\lambda, \mu}-$ функция в правой части (31).

Далее, пусть $F \in \mathscr{D}_{\natural}^{\prime}(-R, R), w \in \mathscr{D}\left(\mathscr{B}_{R}\right)$. Рассмотрим $\eta \in \mathscr{D}_{\sharp}^{\prime}(-R, R)$ такую, что $\eta=1$ на $\left(-r_{0}(w)-\varepsilon, r_{0}(w)+\varepsilon\right)$ при некотором $\varepsilon \in\left(0, R-r_{0}(w)\right)$. Тогда, как и выше, из доказательства леммы 2 и теоремы 2 следует, что равенство

$$
\begin{aligned}
\left\langle\mathfrak{B}_{p, q, l}(F), w\right\rangle & =\frac{1}{\pi} \int_{0}^{\infty} \widehat{F \eta}(\lambda) \mathscr{F}_{l}^{p, q}\left((w)_{(p, q), l}(\varrho) S_{l}^{p, q}(\varrho)\right)(\lambda) d \lambda \\
& =\frac{1}{\pi} \int_{0}^{\infty} \widehat{F \eta}(\lambda)\left\langle w, \phi_{\lambda, 0, p, q, l}\right\rangle d \lambda
\end{aligned}
$$

определяет $\mathfrak{B}_{p, q, l}(F)$ как распределение в $\mathscr{D}_{p, q, l}^{\prime}\left(\mathscr{B}_{R}\right)$, а отображение $\mathfrak{B}_{p, q, l}$ является гомеоморфизмом $\mathscr{D}_{\natural}^{\prime}(-R, R)$ на $\mathscr{D}_{p, q, l}^{\prime}\left(\mathscr{B}_{R}\right)$ и $C_{\natural}^{\infty}(-R, R)$ на $C_{p, q, l}^{\infty}\left(\mathscr{B}_{R}\right)$. Кроме того, $\mathfrak{B}_{p, q, l}^{-1}=\mathfrak{A}_{p, q, l}$.

Построенные операторы позволяют получить в случае групп Гейзенберга аналоги ряда евклидовых результатов для уравнений свертки из [4; часть 3]. Мы остановимся более детально на аналогах теорем 6 и 7.

Пусть $T$ - распределение с компактным носителем на $H_{\mathrm{red}}^{n}$. Положим

$$
r(T)=\inf \left\{r>0: \operatorname{supp} T \subset C_{r}\right\}
$$


где

$$
C_{r}=\left\{\{(z, t+2 \pi k): k \in \mathbb{Z}\} \in H_{\text {red }}^{n}: z \in \mathscr{B}_{r}, 0 \leqslant t<2 \pi\right\} .
$$

Для $f \in \mathscr{D}^{\prime}\left(C_{r}\right)$ определим $f_{k}=\left\langle f, e^{-i k t}\right\rangle, k \in \mathbb{Z}$. Звездочкой * обозначим свертку на группе $H_{\text {red }}^{n}$. Пусть также $\zeta_{a}(a>0)$ - оператор растяжения на $\mathbb{C}^{n}$, переводящий распределение $f \in \mathscr{D}^{\prime}\left(\mathbb{C}^{n}\right)$ в распределение $f(a z)$.

Теорема 10. Пусть $\left\{T_{\alpha}\right\}_{\alpha \in A}$ - семейство распределений с компактными носителями на $H_{\mathrm{red}}^{n}$, которые являются радиальными по переменной $z$. Предположим, что $r\left(T_{\nu}\right)+\inf _{\alpha \in A} r\left(T_{\alpha}\right)<R \leqslant+\infty$ для любого $\nu \in A u$

$$
\bigcup_{k \in \mathbb{Z} \backslash\{0\}} \bigcap_{\alpha \in A} \mathscr{Z}\left(\mathscr{F}_{1}^{0,0}\left(\zeta_{1 / \sqrt{|k|}}\left(T_{\alpha}\right)_{k}\right)\right)=\bigcap_{\alpha \in A} \mathscr{Z}\left(\widetilde{\left(T_{\alpha}\right)_{0}}\right)=\varnothing,
$$

где $\widetilde{\left(T_{\alpha}\right)_{0}}-$ евклидово сферическое преобразование распределения $\left(T_{\alpha}\right)_{0}$ на $\mathbb{C}^{n}$.

Тогда

$$
\left\{f \in \mathscr{D}^{\prime}\left(C_{R}\right): f * T_{\alpha}=0 \text { в } C_{R-r\left(T_{\alpha}\right)} \forall \alpha \in A\right\}=\{0\} .
$$

Если $\sup _{\alpha \in A} r\left(T_{\alpha}\right)<+\infty$, то сформулированное выше утверждение справедливо и при $R=\inf _{\alpha \in A} r\left(T_{\alpha}\right)+\sup _{\alpha \in A} r\left(T_{\alpha}\right)$.

ДоказАтельство. Пусть $f \in \mathscr{D}^{\prime}\left(C_{R}\right)$. Нетрудно видеть, что

$$
\left(f * T_{\alpha}\right)_{k}=f_{k} \times_{-k}\left(T_{\alpha}\right)_{k} \quad \text { в } \mathscr{B}_{R-r\left(T_{\alpha}\right)},
$$

где распределение в правой части $\left((-k)\right.$-искаженная свертка $f_{k}$ и $\left.\left(T_{\alpha}\right)_{k}\right)$ определяется равенством

$$
\begin{gathered}
\left\langle f_{k} \times_{-k}\left(T_{\alpha}\right)_{k}, \psi\right\rangle=\left\langle f_{k}(z),\left\langle\left(T_{\alpha}\right)_{k}(w), \psi(z+w) e^{-i k \operatorname{Im}\langle z, w\rangle_{\mathbb{C}} / 2}\right\rangle\right\rangle=0, \\
\psi \in \mathscr{D}\left(\mathscr{B}_{R-r\left(T_{\alpha}\right)}\right) .
\end{gathered}
$$

Применяя теперь теорему 6 в одномерном случае, (23) и утверждение (i) теоремы 9 , получаем требуемое утверждение.

СлЕДСТВИЕ 6. Пусть $\left\{T_{\alpha}\right\}_{\alpha \in A}$ - семейство радиальных распределений из $\mathscr{E}^{\prime}\left(\mathbb{C}^{n}\right)$. Предположим, что $r\left(T_{\nu}\right)+\inf _{\alpha \in A} r\left(T_{\alpha}\right)<R \leqslant+\infty$ для любого $\nu \in A$ $u \bigcap_{\alpha \in A} \mathscr{Z}\left(\mathscr{F}_{1}^{0,0}\left(T_{\alpha}\right)\right)=\varnothing$.

Тогда

$$
\left\{f \in \mathscr{D}^{\prime}\left(\mathscr{B}_{R}\right): f \times T_{\alpha}=0 \text { в } \mathscr{B}_{R-r\left(T_{\alpha}\right)} \forall \alpha \in A\right\}=\{0\} .
$$

Если $\sup _{\alpha \in A} r\left(T_{\alpha}\right)<+\infty$, то сформулированное выше утверждение справедливо и при $R=\inf _{\alpha \in A} r\left(T_{\alpha}\right)+\sup _{\alpha \in A} r\left(T_{\alpha}\right)$.

ДоказАтельство. Учитывая соотношение (95) при $k=-1$, заключаем, что достаточно использовать теорему 10 для распределений вида $T(z) e^{-i t}$.

Одним из приложений теоремы 10 является

Tеорема 11. (i) Пусть $V$ - ненулевое подпространство в $C\left(H_{\text {red }}^{n}\right)$, инвариантное относительно групповых сдвигов и действия унитарной группы $U(n)$.

Тогда $V$ содержит хотя бъ одну из функиий

$$
(\lambda|z|)^{-n+1} J_{n-1}(\lambda|z|), \quad \phi_{\lambda, 0,0,0}(\sqrt{|k|}|z|) e^{i k t}
$$

при некоторых $\lambda \in \mathbb{C}, k \in \mathbb{Z} \backslash\{0\}$. 
(ii) Пусть $V$ - ненулевое подпространство в $C\left(\mathbb{C}^{n}\right)$, инвариантное относительно искаженных сдвигов $\tau(w)$ (cм. §1) и унитарных преобразований.

Тогда подпространство $V$ содержит функцию $\phi_{\lambda, 0,0,0}(|z|)$ при некотором $\lambda \in \mathbb{C}$.

ДоказАтельство. (i) Из теоремы Хана-Банаха следует (см. [12; §4]), что

$$
V=\left\{f \in C\left(H_{\text {red }}^{n}\right): f * T_{\alpha}=0 \forall \alpha \in A\right\}
$$

для некоторого семейства $\left\{T_{\alpha}\right\}_{\alpha \in A}$ ненулевых радиальных распределений на $H_{\text {red }}^{n}$ с компактными носителями. Применяя теорему 10, (80) и теорему о среднем для собственных функций евклидова лапласиана, мы завершаем доказательство (i). Утверждение (ii) доказывается аналогично (см. следствие 6).

ЗАмЕчАниЕ 2. Как уже отмечалось, для подпространств $V$, порождаемых функциями медленного роста, аналоги теоремы 11 были доказаны С. Сангавелу (см. [12; теоремы 4.3, 6.2]). Там же высказана гипотеза относительно общей ситуации. В частности, в случае (ii) теоремы 11 предполагается, что $V$ содержит функцию $f(z)=e^{i[z, \zeta]} L_{k}^{n-1}\left(|z|^{2} / 2\right)$ при некоторых $\zeta \in \mathbb{C}^{2 n}, k \in \mathbb{Z}_{+}$, где $[z, \zeta]$ - симплектическая форма. Однако нетрудно проверить, что $\mathfrak{L} f \neq \lambda f$ для $\lambda \notin n+2 \mathbb{Z}_{+}$. Поэтому инвариантное подпространство $\left\{g \in C\left(\mathbb{C}^{n}\right): \mathfrak{L} g=\lambda g\right\}$, где $\lambda \notin n+2 \mathbb{Z}_{+}$, не содержит $f$. Это опровергает гипотезу С. Сангавелу.

В заключение мы приведем аналог теоремы 7 для искаженной свертки. Пусть $\Psi=\left\{\psi_{\alpha}\right\}_{\alpha \in A}-$ семейство распределений из $\mathscr{E}^{\prime}\left(\mathbb{C}^{n}\right)$ такое, что $r\left(\psi_{\alpha}\right)<$ $R \leqslant+\infty$ для всех $\alpha \in A$. Говорят, что семейство $\Psi=\left\{\psi_{\alpha}\right\}_{\alpha \in A}$ имеет искаженное свойство Помпейю в шаре $\mathscr{B}_{R}$, если не существует ненулевой функции $f \in C^{\infty}\left(\mathscr{B}_{R}\right)$ такой, что

$$
(f \circ \tau) \times \psi_{\alpha}=0 \quad \text { в } \mathscr{B}_{R-r\left(\psi_{\alpha}\right)} \text { для всех } \tau \in U(n), \alpha \in A .
$$

ТЕОРема 12. Пусть $\Psi=\left\{\psi_{\alpha}\right\}_{\alpha \in A}$ - семейство распределений класса $\mathscr{E}^{\prime}\left(\mathbb{C}^{n}\right)$ таких, что $\sup _{\alpha} r\left(\psi_{\alpha}\right)<+\infty$. Тогда при $R>\inf _{\alpha} r\left(\psi_{\alpha}\right)+\sup _{\alpha} r\left(\psi_{\alpha}\right)$ следующие утверждения эквивалентны:

(i) семейство $\Psi$ имеет искаженное свойство Помпейю в шаре $\mathscr{B}_{R}$;

(ii) семейство $\Psi$ имеет искаженное свойство Помпейю в $\mathbb{C}^{n}$.

ДокАЗАТЕЛЬСтво. Повторяя рассуждения в доказательстве теоремы 7 (но теперь используя (60), (80), утверждение (viii) предложения 1 и следствие 6), получаем требуемый результат.

\section{Список литературы}

[1] К. Беренстейн, Д. Струппа, "Комплексный анализ и уравнения в свертках", Комплексный анализ. Многие переменные - 5, Итоги науки и техн. Сер. Соврем. пробл. матем. Фундам. направления, 54, ВИНИТИ, М., 1989, 5-111; англ. пер.: C. A. Berenstein, D. C. Struppa, Several complex variables. V. Complex analysis in partial differential equations and mathematical physics, Encyclopaedia Math. Sci., 54, Springer-Verlag, Berlin, 1993. 
[2] L. Brown, B. M. Schreiber, B. A. Taylor, "Spectral synthesis and the Pompeiu problem", Ann. Inst. Fourier (Grenoble), 23:3 (1973), 125-154.

[3] Д. И. Гуревич, "Контрпримеры к проблеме Л. Шварца", Функи. анализ и его прил., 9:2 (1975), 29-35; англ. пер.: D. I. Gurevich, "Counterexamples to a problem of L. Schwartz", Funct. Anal. Appl., 9:2 (1975), 116-120.

[4] V.V. Volchkov, Integral geometry and convolution equations, Kluwer Acad. Publ., Dordrecht, 2003.

[5] C. A. Berenstein, R. Gay, "A local version of the two-circles theorem", Israel J. Math., $\mathbf{5 5 : 3}$ (1986), 267-288.

[6] Л. Хёрмандер, Анализ линейных дифференциальных операторов с частными производными, т. 1, 2, Мир, М., 1986; пер. с англ.: L. Hörmander, The analysis of linear partial differential operators, vols. 1, 2, Grundlehren Math. Wiss., 256-257, Springer-Verlag, Berlin, 1983.

[7] В. В. Напалков, Уравнения свертки в многомерных пространствах, Наука, М., 1982.

[8] G. B. Folland, Harmonic analysis in phase space, Ann. of Math. Stud., 122, Princeton Univ. Press, Princeton, NJ, 1989.

[9] S. Thangavelu, Lectures on Hermite and Laguerre expansions, Math. Notes, 42, Princeton Univ. Press, Princeton, NJ, 1993.

[10] Л.А. Айзенберг, А.П. Южаков, Интегралънъе представления и вычеты в многомерном комплексном анализе, Наука, Новосибирск, 1979; англ. пер.: L. A. Aǐzenberg, A.P. Yuzhakov, Integral representations and residues in multidimensional complex analysis, Transl. Math. Monogr., 58, Amer. Math. Soc., Providence, RI, 1983.

[11] S. Thangavelu, "Spherical means and CR functions on the Heisenberg group", J. Anal. Math., 63 (1994), 255-286.

[12] S. Thangavelu, "Mean periodic functions on phase space and the Pompeiu problem with a twist", Ann. Inst. Fourier (Grenoble), 45:4 (1995), 1007-1035.

[13] C. A. Berenstein, R. Gay, "Le problème de Pompeiu local", J. Analyse Math., 52:1 (1981), 133-166.

[14] А.Ф. Леонтьев, Последовательности полиномов из экспонент, Наука, М., 1980.

[15] И. Стейн, Г. Вейс, Введение в гармонический анализ на евклидовых пространствах, Мир, М., 1974; пер. с англ.: Е. M. Stein, G. Weiss, Introduction to Fourier analysis on Euclidean spaces, Princeton Math. Ser., 32, Princeton Univ. Press, Princeton, NJ, 1971.

[16] Н. Я. Виленкин, Специальные функиии и теория представлений групп, 2-е изд., Наука, М., 1991; англ. пер. 1-го изд.: N. Ya. Vilenkin, Special functions and the theory of group representations, Transl. Math. Monogr., 22, Amer. Math. Soc., Providence, RI, 1968.

[17] Г. Бейтмен, А. Эрдейи, Высшие трансиендентные функиии, т. 1, 2, Наука, М., 1973-1974; пер. с англ.: A. Erdélyi, W. Magnus, F. Oberhettinger, F. G. Tricomi, Higher transcendental functions, vols. I, II, McGraw-Hill, New York-Toronto-London, 1953.

[18] У. Рудин, Теория функиий в единичном шаре из $\mathbb{C}^{n}$, Мир, М., 1984; пер. с англ.: W. Rudin, Function theory in the unit ball of $\mathbb{C}^{n}$, Grundlehren Math. Wiss., 241, Springer-Verlag, New York-Berlin, 1980.

[19] R. A. Askey, T.H. Koornwinder, W. Schempp (eds.), Special functions: group theoretical aspects and applications, Math. Appl., 18, Reidel Publ., DordrechtBoston-Lancaster, 1984. 
[20] С. Хелгасон, Группы и геометрический анализ, Мир, М., 1987; пер. с англ.: S. Helgason, Groups and geometric analysis, Pure Appl. Math., 113, Academic Press, Orlando, FL, 1984.

В. В. Волчков (V. V. Volchkov)

Донецкий национальный университет, Украина

E-mail: valeriyvolchkov@gmail.com

Вит. В. Волчков (Vit. V. Volchkov)

Донецкий национальный университет, Украина

E-mail: volchkov@univ.donetsk.ua
Поступила в редакцию 12.09.2007 и 21.03.2008 